\title{
Analysis of Nonlinear Vibrations and Dynamic Responses in a Trapezoidal Cantilever Plate Using the Rayleigh-Ritz Approach Combined with the Affine Transformation
}

\author{
Wei Tian $\mathbb{D}^{1,2}$ Zhichun Yang, $^{3}$ and Tian Zhao ${ }^{2}$ \\ ${ }^{1}$ School of Aerospace, Xian Jiaotong University, Xian, China \\ ${ }^{2}$ National Key Laboratory of Science and Technology on Liquid Rocket Engine, Xian Aerospace Propulsion Institute, Xian, China \\ ${ }^{3}$ School of Aeronautics, Northwestern Polytechnical University, Xian, China
}

Correspondence should be addressed to Wei Tian; twtp100@163.com

Received 28 April 2019; Accepted 4 July 2019; Published 25 July 2019

Academic Editor: Nhon Nguyen-Thanh

Copyright (C) 2019 Wei Tian et al. This is an open access article distributed under the Creative Commons Attribution License, which permits unrestricted use, distribution, and reproduction in any medium, provided the original work is properly cited.

Nonlinear vibrations of a trapezoidal cantilever plate subjected to transverse external excitation are investigated. Based on von Karman large deformation theory, the Rayleigh-Ritz approach combined with the affine transformation is developed to obtain the nonlinear ordinary differential equation of a trapezoidal plate with irregular geometries. With the variation of geometrical parameters, there exists the 1:3 internal resonance for the trapezoidal plate. The amplitude-frequency formulations of the system in three different coupled conditions are derived by using multiple scales method for 1:3 internal resonance analysis. It is found that the strong coupling of two modes can change nonlinear stiffness behaviors of modes from hardening-spring to soft-spring characteristics. The detuning parameter and excitation amplitude have significant influence on nonlinear dynamic responses of the system. The bifurcation diagrams show that there exist the periodic, quasi-periodic, and chaotic motions for the trapezoidal cantilever plate in the 1:3 internal resonance cases and the nonlinear dynamic responses are dependent on the amplitude of excitation. The possible adverse dynamic behaviors and undesired resonance can be avoided by designing appropriate excitation and system parameters.

\section{Introduction}

The linear and nonlinear vibration analysis of plate-like structures has been paid much attention in mechanical, civil, and aerospace engineering fields. The understanding of the vibration behaviors is critical in many aspects, e.g., plate/panel flutter [1-3], energy harvesting of flexible plates [4], and nonlinear aeroelastic responses of functionally graded materials (FGM) plates, as well as the coupling of these structures with electro-magnetic, acoustic, and thermal fields $[5,6]$. Structural nonlinearity exists unavoidably in the engineering structures, which significantly affects the nonlinear oscillation behavior and flutter instability [7]. Consequently, the nonlinear vibration analysis can provide theoretical significance for ground vibration test and flutter analysis of flight vehicle structures.
The nonlinear vibration behaviors of plate-like structures have been investigated by many researchers. The nonlinear vibration of the rectangular plate under the transverse and in-plane excitations was analyzed by Hao et al. [8] in 2008. It was found that periodic, quasi-period, and chaotic responses for the rectangular plate models take place under certain conditions. Later on, Hao et al. [9] investigated the nonlinear dynamic behaviors of cantilever FGM rectangular plates under combined transverse excitation force and thermal loads. Their studies demonstrated that the nonlinear dynamic response of the cantilever plate is rather sensitive to transverse external excitation compared with that of a simply supported plate. Zhang [10] studied the bifurcations and chaotic characteristics of a simply supported rectangular plate under a parametric excitation by using the multiple scales method. The results showed that the rectangular plate can undergo Hopf bifurcation, heteroclinic bifurcations, 
and Shilnikov-type homoclinic orbit to the saddle focus. Meanwhile, Zhang and Liu [11] analyzed the local and global bifurcations of the same model subjected to transverse and in-plane excitations simultaneously. The chaotic responses obtained by the numerical simulation are sensitive to the initial conditions. An and Chen [12] investigated the global bifurcation and chaotic behaviors of a simply supported FGM plate. They also found that the types of the chaotic motions are completely different under different excitations, system parameters, and initial values. Mousa and Reza [13] studied the nonlinear dynamics and chaotic behaviors of a simply supported plate under in-plane/transverse excitations and aerodynamic loading, and the effects of detuning parameter, excitation amplitude, and dynamic pressure on the nonlinear dynamics of the model were analyzed. It was also found that the variation of control parameters can lead to the multiperiodic and chaotic motions. Sayed et al. [14] studied the nonlinear vibration of a two degree-of-freedom (DOFs) system with quadratic and cubic nonlinearities subjected to external and parametric excitation forces. Their results showed that all possible resonance could be observed and the system also exhibited chaotic motions under certain conditions.

Generally, in order to solve the nonlinear governing equations of panel/plate efficiently, the Rayleigh-Ritz or Galerkin approach is used for the modal representation of transverse deflections, and the obtained ordinary differential equations (ODEs) can be further solved in modal coordinates analytically and numerically. Ye et al. [15] used RayleighRitz approach to obtain the limit cycle oscillation (LCO) of a cantilever plate and discussed the effect of length-to-width ratio on the nonlinear oscillations of the system. Later on, Xie et al. [16] extended the work of Ye and Dowell and used the same model to examine the chaotic motions and the routes to chaos with the increasing of dynamic pressure. The bifurcation behaviors of a simply supported square plate with the airflow and structural nonlinearities were also studied, and they evaluated the effect of the aerodynamic nonlinearity [17]. Bakhtiari-Nejad and Nazari [18] analyzed the nonlinear vibration of a cantilever plate with viscoelastic laminate by Rayleigh-Ritz method. The stability and chaotic behavior were obtained by using numerical simulations. Liew et al. [19] discussed the nonlinear vibration behavior of cantilever plates with constant thickness based on the Mindlin plate theory. Yang et al. [20] studied the nonlinear response characteristics and experiments of a cantilever hard-coating plate under transverse external excitation. The vibration response experiment agreed well with the numerical results. Dai et al. [21-23] proposed a highly efficient global nonlinear Galerkin method for the analysis of the large-deflection problem of plates. Additionally, a time domain collocation method also has been proposed by Dai et al. [24, 25] to solve nonlinear oscillatory problems, which is promising in the analysis of the von Karman fluttering plate. The aforementioned studies applied the Galerkin/Rayleigh-Ritz approaches to the modelling of nonlinear systems, which could achieve the purposes of DOF reduction and computational cost saving, compared with traditional finite element method.
For nonlinear vibration analysis, due to the complexity for obtaining analytical solutions of nonlinear differential equations, many asymptotic approaches have been developed for this purpose, such as energy balance method [26], variational approach [27], multiple scales method [28-32], and homotopy perturbation method. Here, the multiple scales method is a very powerful and well-known approach for analyzing the primary, internal, and super/subharmonic and higherorder resonances of nonlinear dynamic systems by several researchers. Ye et al. [29] utilized the multiple scales method to obtain the nonlinear oscillations and chaotic behaviors of an antisymmetric cross-ply laminated composite rectangular thin plate under parametric excitation. Their results showed the multipulse orbits exist in the present model. Sayed and Mousa [30] used this approach to analyze the nonlinear dynamic behaviors of the composite laminated plate with transverse excitations. The investigation demonstrated that the variation of system parameters could avoid worst resonance cases. Zhang et al. [31] used the multiple scales method to obtain the averaged equation of cantilever rectangular plate under in-plane force and moment for nonlinear vibration analysis and their studies showed that nonlinear dynamic responses could be controlled by changing these two kinds of excitations. Alijani et al. [32] studied the bifurcations and complicated nonlinear dynamics of FGM rectangular plates based on the von Karman's nonlinear geometry plate theory and the multiple scales method subjected to the transverse excitation in thermal environment. It was found that the thermally deformed FGM plates have a strong hardening behavior and the effect of volume fraction exponent on nonlinear behavior is not significant. Later on, they analyzed nonlinear parametric instability of FGM plates in thermal environments and showed the complex nonlinear dynamic responses through period-doubling and NeimarkSacker bifurcations [33]. Xue et al. [34] analyzed the nonlinear principal resonances of an orthotropic magnetoelastic plate under a transverse magnetic field and a harmonic mechanical force using the multiple scales method. It was found that the nonlinear hardening effect of the principal resonance for the isotropic plate is much stronger than that of the corresponding orthotropic plate.

Due to the simply geometries commonly used in engineering structures and the limitation of the traditional methods, the aforementioned literatures in this field indicate that most of the studies are dealing with nonlinear dynamic problems of rectangle plates. Few studies have been performed on nonlinear dynamic behaviors of the cantilever plates with irregular geometries. Meanwhile, cantilever-like structures are commonly used in a large number of engineering fields such as engine blades, fixed wings, and helicopter rotor blades in aerospace engineering. The studies of nonlinear dynamic behaviors of cantilever plates provide useful insight into ground vibration tests and structure dynamic designs. To take a low-aspect-ratio wing as an example, it can be simplified as a cantilevered trapezoidal wing-like plate. Recently, the nonlinear dynamic characteristics of a trapezoidal wing model under subsonic or hypersonic aerodynamic loads were also investigated by Shokrollahi et al. [35] and Tian et al. [36]. 


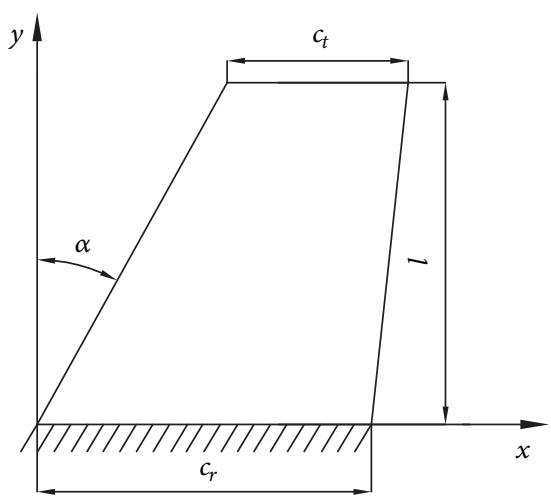

(a)

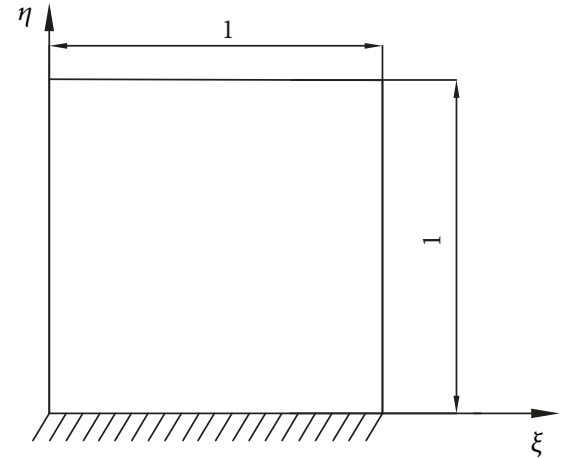

(b)

FIGURE 1: (a) Schematic of trapezoidal cantilever plate geometry; (b) the transformed nondimensional square plate model.

Besides, it is also noted that most studies focus on nonlinear vibrations of rectangular plate, and there exist certain resonances with the variations of physical parameters of composite or FGM material. However, to the authors' knowledge, the studies of parametric resonance and complex dynamic responses of the trapezoidal cantilever plates with irregular geometries have not been explored. Therefore, nonlinear vibration behaviors of a trapezoidal cantilever plate subjected to transverse external excitation are explored in the current study and focused on the 1:3 internal resonance of the plate. The contents of the work are organized as follows. In Section 2, the affine transformation combined with the Rayleigh-Ritz method is used to obtain the nonlinear vibration equations of trapezoidal cantilever plate. Section 3 establishes the averaged equations of the transverse motion by using the multiple scales method. Section 4 is devoted to the studies on the amplitude-frequency characteristics of the system with the 1:3 internal resonance. In Section 5, the nonlinear dynamic responses under certain conditions are obtained numerically from the derived averaged equations. The main conclusions are drawn in Section 6.

\section{Mathematical Modeling}

A trapezoidal cantilever plate shown in Figure 1(a) is constrained along the $y=0$ edge. The parameters $c_{r}, c_{t}, l$, and $\alpha$ are defined in Figure 1(a). The nonlinear vibration equations are obtained from Lagrange's equations based on the von Karman nonlinear strain-displacement relation using kinetic and potential energies.

The kinetic energy $T$ and potential energy $U$ can be expressed as

$$
\begin{aligned}
& T=\frac{1}{2} \iint \rho_{m} h\left(\frac{\partial w}{\partial t}\right)^{2} d x d y \\
& U=U_{S}+U_{B}
\end{aligned}
$$

where $U_{S}$ and $U_{B}$ are the potential energies induced by stretching and bending, respectively. Their expressions can be given as

$$
\begin{aligned}
U_{S} & =\frac{E h}{2\left(1-v^{2}\right)} \iint\left\{\left[\frac{\partial u}{\partial x}+\frac{1}{2}\left(\frac{\partial^{2} w}{\partial x^{2}}\right)\right]^{2}\right. \\
& +\left[\frac{\partial v}{\partial y}+\frac{1}{2}\left(\frac{\partial^{2} w}{\partial y^{2}}\right)\right]^{2}+2 v\left[\frac{\partial u}{\partial x}+\frac{1}{2}\left(\frac{\partial^{2} w}{\partial x^{2}}\right)\right] \\
& \cdot\left[\frac{\partial v}{\partial y}+\frac{1}{2}\left(\frac{\partial^{2} w}{\partial y^{2}}\right)\right]+\frac{1-v}{2}\left[\frac{\partial v}{\partial x}+\frac{\partial u}{\partial y}\right. \\
& \left.\left.+\frac{\partial w}{\partial x} \frac{\partial w}{\partial y}\right]^{2}\right\} d x d y \\
U_{B} & =\frac{D}{2} \iint\left[\left(\frac{\partial^{2} w}{\partial x^{2}}\right)^{2}+\left(\frac{\partial^{2} w}{\partial y^{2}}\right)^{2}+2 \nu \frac{\partial^{2} w}{\partial x^{2}} \frac{\partial^{2} w}{\partial y^{2}}\right. \\
& \left.+2(1-v)\left(\frac{\partial^{2} w}{\partial x \partial y}\right)^{2}\right] d x d y
\end{aligned}
$$

The Lagrange's equation is

$$
\frac{d}{d t}\left(\frac{\partial L}{\partial \dot{q}_{m n}}\right)-\frac{\partial L}{\partial q_{m n}}+Q_{m n}=0
$$

where $L=T-U$ is the Lagrangian and $Q_{m n}$ is the generalized force.

Substituting the expressions of kinetic and potential energy into (3), one can obtain

$$
\frac{d}{d t}\left(\frac{\partial T}{\partial \dot{q}_{m n}}\right)+\frac{\partial U_{B}}{\partial q_{m n}}+\frac{\partial U_{S}}{\partial q_{m n}}+Q_{m n}=0
$$

The Rayleigh-Ritz method is used to transform the equations of a trapezoidal plate model into modal coordinates, and the ordinary beam mode functions can be used as the 
mode functions of the trapezoidal plate. Then, the nondimensional deflections in $\xi$, $\eta$, and $z$ directions are expressed as

$$
\begin{aligned}
& \bar{u}=\sum_{i=1}^{I} \sum_{j=1}^{J} a_{i j}(\tau) u_{i}(\xi) v_{j}(\eta) \\
& \bar{v}=\sum_{r=1}^{R} \sum_{s=1}^{S} b_{r s}(\tau) u_{r}(\xi) v_{s}(\eta)
\end{aligned}
$$

$$
\begin{aligned}
& u_{i}(\xi)=\cos i \pi \xi \\
& v_{j}(\eta)=\sin \frac{2 j-1}{2} \pi \xi \\
& u_{r}(\xi)=\cos r \pi \xi \\
& v_{s}(\eta)=\sin \frac{2 s-1}{2} \pi \xi \\
& \phi_{m}(\xi)=\left\{\begin{array}{lr}
2 & m=1 \\
2(1-\xi) & m=2 \\
\sqrt{2} \sin \left(\beta_{m} \xi+\frac{3 \pi}{4}\right)+\exp \left(-\beta_{m} \xi\right)+(-1)^{m+1} \exp \left[-\beta_{m}(1-\xi)\right] & m>2
\end{array}\right. \\
& \psi_{n}(\eta)=\sqrt{2} \sin \left(\beta_{n} \eta-\frac{\pi}{4}\right)+\exp \left(-\beta_{n} \eta\right)+(-1)^{n+1} \exp \left[-\beta_{n}(1-\eta)\right]+(-1)^{n} \exp \left(-\beta_{n}\right)
\end{aligned}
$$

with $\beta_{m}=(m-3 / 2) \pi, \beta_{n}=(n-1 / 2) \pi$, where $\phi_{m}(\xi)$ and $\psi_{n}(\eta)$ are the mode functions of free-free beam and cantilever beam, respectively.

For the trapezoidal cantilever plate, the mode functions of free-free beam and cantilever beam cannot be used directly. The trapezoidal plate model can be mapped into a nondimensional square plate by using the affine transformation [36], which is shown in Figure 1(b). The affine transformation can be given as

$$
\begin{aligned}
& \xi=\frac{x / c_{r}-(A R / 4)(1+T R) \tan \alpha(y / l)}{1-(1-T R)(y / l)}, \\
& \eta=\frac{y}{l}
\end{aligned}
$$

where TR $\left(=c_{t} / c_{r}\right)$ is defined as the ratio of $c_{t}$ and $c_{r}$ and $A R$ is defined in terms of $c_{r}, l$, and $T R$ as $A R=4\left(l / c_{r}\right) /(1+T R)$.

Accordingly, the transformation matrix $\mathbf{J}$ can be obtained as

$$
\begin{aligned}
\mathbf{J} & =\left[\begin{array}{ll}
\frac{\partial x}{\partial \xi} & \frac{\partial x}{\partial \eta} \\
\frac{\partial y}{\partial \xi} & \frac{\partial y}{\partial \eta}
\end{array}\right] \\
& =\left[\begin{array}{cc}
c_{r}[1-(1-T R) \eta] & \frac{A R}{4}(1+T R) \tan \alpha \\
0 & l
\end{array}\right]
\end{aligned}
$$

For the trapezoidal plate model under transverse excitation, which is shown in Figure 2, the transverse nonlinear vibration is dominant; namely, the effects of the in-plane motions $\bar{u}$ and $\bar{v}$ can be ignored, and only transverse vibration of the trapezoidal cantilever plate is considered for the following analysis.

The present study will focus on the case of 1:3 internal resonance of the trapezoidal cantilever plate. Based on the analysis of the existing studies [24-26], the first two modes of nonlinear oscillations are enough to represent the nonlinear vibration behaviors of the plate, especially used for internal resonance analysis. Thus, the nonlinear oscillations of the trapezoidal cantilever plate in the first two modes are considered only. The expression for transverse deflection $\bar{w}$ is given by

$$
\bar{w}=q_{1}(\tau) \phi_{1}(\xi) \psi_{1}(\eta)+q_{2}(\tau) \phi_{2}(\xi) \psi_{1}(\eta)
$$

Substituting (9) into (4) and introducing Rayleigh damping, the nondimensional transverse motion equation subjected to transverse harmonic excitation $P_{z}=P_{0} \cos \Omega_{0} \tau$ can be obtained

$$
\mathbf{A} \ddot{\mathbf{q}}+\mathbf{B q}+\mathbf{F}=\mathbf{P}
$$


Mathematical Problems in Engineering

5

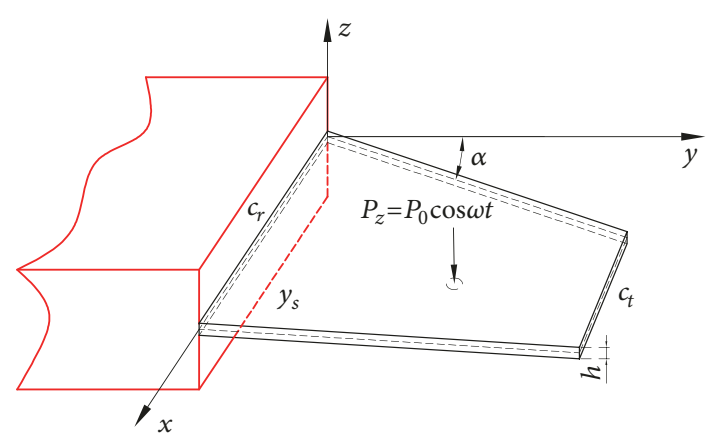

FIGURE 2: A trapezoidal cantilever plate subjected to transverse harmonic excitation.

where

$$
\begin{aligned}
\mathbf{q} & =\left[\begin{array}{l}
q_{1} \\
q_{2}
\end{array}\right], \\
\mathbf{A} & =\left[\begin{array}{ll}
A_{11} & A_{12} \\
A_{21} & A_{22}
\end{array}\right], \\
\mathbf{B} & =\left[\begin{array}{ll}
B_{11} & B_{12} \\
B_{21} & B_{22}
\end{array}\right], \\
\mathbf{F} & =\left[\begin{array}{l}
F_{1} \\
F_{2}
\end{array}\right], \\
\mathbf{P} & =\left[\begin{array}{l}
P_{1} \\
P_{2}
\end{array}\right] \cos \Omega_{0}, \\
F_{1} & =F_{q_{1}^{3}, 1} q_{1}^{3}+F_{q_{1}^{2} q_{2}, 1} q_{1}^{2} q_{2}+F_{q_{1} q_{2}^{2}, 1} q_{1} q_{2}^{2}+F_{q_{2}^{3}, 1} q_{2}^{3}, \\
F_{2} & =F_{q_{2}^{3}, 1} q_{2}^{3}+F_{q_{2} q_{1}^{2}, 1} q_{1} q_{2}^{2}+F_{q_{1}^{2} q_{2}, 1} q_{1}^{2} q_{2}+F_{q_{1}^{3}, 1}^{3}, \\
P_{1} & =P_{0} \varphi_{1}\left(\xi_{e}\right) \psi_{1}\left(\eta_{e}\right), \\
P_{2} & =P_{0} \varphi_{2}\left(\xi_{e}\right) \psi_{1}\left(\eta_{e}\right) .
\end{aligned}
$$

(11)

The element expressions of matrices A, B are given in (12a) and (12b), and the coefficients of $F_{q_{1}^{3}, i}, F_{q_{1}^{2} q_{2}, i}, F_{q_{1} q_{2}^{2}, i}$, and $F_{q_{2}^{3}, i}(i=1,2)$ are given in the Appendix.

$$
\begin{aligned}
& A_{i j}=\frac{1}{6} \int_{0}^{1} \phi_{i} \phi_{j} d \xi \int_{0}^{1} \psi_{1} \psi_{1} J^{\prime} d \eta \\
& B_{i j}=\frac{1}{6}\left\{\int_{0}^{1} \phi_{i}^{\prime \prime} \phi_{j}^{\prime \prime} d \xi \int_{0}^{1} \psi_{1} \psi_{1} H^{4} J^{\prime} d \eta+\left(\frac{c_{r}}{l}\right)^{2}\right. \\
& \cdot {\left[2 \nu \int_{0}^{1} \phi_{i}^{\prime \prime} \phi_{j}^{\prime \prime} G^{2} d \xi \int_{0}^{1} \psi_{1} \psi_{1} H^{4} J^{\prime} d \eta+2(1-\nu)\right.} \\
& \cdot \int_{0}^{1} \phi_{i}^{\prime \prime} \phi_{j}^{\prime \prime} G^{2} d \xi \int_{0}^{1} \psi_{1} \psi_{1} H^{4} J^{\prime} d \eta+2(1-\nu) \\
& \cdot c_{2}^{2} \int_{0}^{1} \phi_{i}^{\prime} \phi_{j}^{\prime} d \xi \int_{0}^{1} \psi_{1} \psi_{1} H^{4} J^{\prime} d \eta+2(1-\nu)
\end{aligned}
$$

$$
\begin{aligned}
& \cdot \int_{0}^{1} \phi_{i}^{\prime} \phi_{j}^{\prime} d \xi \int_{0}^{1} \psi_{1}^{\prime} \psi_{1}^{\prime} H^{2} J^{\prime} d \eta+2(1-\nu) \\
& \cdot c_{2} \int_{0}^{1} \phi_{i}^{\prime} \phi_{j}^{\prime} d \xi \int_{0}^{1} \psi_{1} \psi_{1}^{\prime} H^{4} J^{\prime} d \eta+2(1-\nu) \\
& \cdot c_{2} \int_{0}^{1} \phi_{i}^{\prime} \phi_{j}^{\prime} d \xi \int_{0}^{1} \psi_{1}^{\prime} \psi_{1} H^{4} J^{\prime} d \eta+2 c_{2} \int_{0}^{1} \phi_{i}^{\prime} \phi_{j}^{\prime \prime} G d \xi \\
& \text { · } \int_{0}^{1} \psi_{1} \psi_{1} H^{4} J^{\prime} d \eta+2 c_{2} \int_{0}^{1} \phi_{i}^{\prime \prime} \phi_{j}^{\prime} G d \xi \\
& \cdot \int_{0}^{1} \psi_{1} \psi_{1} H^{4} J^{\prime} d \eta+2 \int_{0}^{1} \phi_{i}^{\prime} \phi_{j}^{\prime \prime} G d \xi \\
& \cdot \int_{0}^{1} \psi_{1} \psi_{1}^{\prime} H^{3} J^{\prime} d \eta+2 \int_{0}^{1} \phi_{i}^{\prime \prime} \phi_{j}^{\prime} G d \xi \\
& \cdot \int_{0}^{1} \psi_{1}^{\prime} \psi_{1} H^{3} J^{\prime} d \eta+v \int_{0}^{1} \phi_{i}^{\prime \prime} \phi_{j} d \xi \int_{0}^{1} \psi_{1}^{\prime \prime} \psi_{1} H^{2} J^{\prime} d \eta \\
& \left.+v \int_{0}^{1} \phi_{i}^{\prime \prime} \phi_{j} d \xi \int_{0}^{1} \psi_{1}^{\prime \prime} \psi_{1} H^{2} J^{\prime} d \eta\right] \\
& +\left(\frac{c_{r}}{l}\right)^{4}\left[\int_{0}^{1} \phi_{i}^{\prime \prime} \phi_{j}^{\prime \prime} G^{4} d \xi \int_{0}^{1} \psi_{1} \psi_{1} H^{4} J^{\prime} d \eta\right. \\
& +\int_{0}^{1} \phi_{i} \phi_{j} d \xi \int_{0}^{1} \psi_{1}^{\prime \prime} \psi_{1}^{\prime \prime} J^{\prime} d \eta+\left(2 c_{2}\right)^{2} \int_{0}^{1} \phi_{i}^{\prime} \phi_{j}^{\prime} G^{2} d \xi \\
& \cdot \int_{0}^{1} \psi_{1} \psi_{1} H^{4} J^{\prime} d \eta \int_{0}^{1} \phi_{i} \phi_{j}^{\prime \prime} G^{2} d \xi \int_{0}^{1} \psi_{1} \psi_{1}^{\prime \prime} H^{2} J^{\prime} d \eta \\
& +2 c_{2} \int_{0}^{1} \phi_{i}^{\prime} \phi_{j}^{\prime \prime} G^{3} d \xi \int_{0}^{1} \psi_{1} \psi_{1} H^{4} J^{\prime} d \eta \\
& +2 c_{2} \int_{0}^{1} \phi_{i}^{\prime \prime} \phi_{j}^{\prime} G^{3} d \xi \int_{0}^{1} \psi_{1} \psi_{1} H^{4} J^{\prime} d \eta \\
& +2 \int_{0}^{1} \phi_{i}^{\prime} \phi_{j}^{\prime \prime} G^{3} d \xi \int_{0}^{1} \psi_{1} \psi_{1}^{\prime} H^{3} J^{\prime} d \eta \\
& +2 \int_{0}^{1} \phi_{i}^{\prime \prime} \phi_{j}^{\prime} G^{3} d \xi \int_{0}^{1} \psi_{1}^{\prime} \psi_{1} H^{3} J^{\prime} d \eta \\
& +4 c_{2} \int_{0}^{1} \phi_{i}^{\prime} \phi_{j}^{\prime} G^{2} d \xi \int_{0}^{1} \psi_{1} \psi_{1}^{\prime} H^{3} J^{\prime} d \eta
\end{aligned}
$$




$$
\begin{aligned}
& +4 c_{2} \int_{0}^{1} \phi_{i}^{\prime} \phi_{j}^{\prime} G^{2} d \xi \int_{0}^{1} \psi_{1}^{\prime} \psi_{1} H^{3} J^{\prime} d \eta \\
& +4 \int_{0}^{1} \phi_{i}^{\prime} \phi_{j}^{\prime} G^{2} d \xi \int_{0}^{1} \psi_{1}^{\prime} \psi_{1}^{\prime} H^{2} J^{\prime} d \eta+\int_{0}^{1} \phi_{i}^{\prime \prime} \phi_{j} G^{2} d \xi \\
& \cdot \int_{0}^{1} \psi_{1}^{\prime \prime} \psi_{1} H^{2} J^{\prime} d \eta+2 c_{2} \int_{0}^{1} \phi_{i}^{\prime} \phi_{j} G d \xi \\
& \cdot \int_{0}^{1} \psi_{1}^{\prime \prime} \psi_{1} H^{2} J^{\prime} d \eta+2 c_{2} \int_{0}^{1} \phi_{i} \phi_{j}^{\prime} G d \xi \\
& \cdot \int_{0}^{1} \psi_{1} \psi_{1}^{\prime \prime} H^{2} J^{\prime} d \eta+\int_{0}^{1} \phi_{i}^{\prime} \phi_{j} G d \xi \int_{0}^{1} \psi_{1}^{\prime \prime} \psi_{1}^{\prime} H J^{\prime} d \eta \\
& \left.\left.+\int_{0}^{1} \phi_{i} \phi_{j}^{\prime} G d \xi \int_{0}^{1} \psi_{1}^{\prime} \psi_{1}^{\prime \prime} H J^{\prime} d \eta\right]\right\}
\end{aligned}
$$

where $H(\eta)=1 /\left(1-c_{2} \eta\right), G(\xi)=c_{2} \xi-c_{1}, J^{\prime}(\eta)=1-c_{2} \eta$, $c_{1}=(A R / 4)(1+T R) \tan \alpha$, and $c_{2}=1-T R$.

Due to the affine transformation employed for trapezoidal plate model, the two modes of (9) are coupled. The mode decoupling need to be performed for the induced linear system of the nonlinear system, then (10) can be rewritten as

$$
\begin{aligned}
\ddot{w}_{1} & +\gamma_{1} \dot{w}_{1}+\omega_{1}^{2} w_{1}+f_{1} w_{1}^{3}+f_{2} w_{1}^{2} w_{2}+f_{3} w_{1} w_{2}^{2} \\
& +f_{4} w_{2}^{3}=k_{1} \cos \Omega_{0} \tau \\
\ddot{w}_{2} & +\gamma_{2} \dot{w}_{2}+\omega_{2}^{2} w_{2}+f_{5} w_{2}^{3}+f_{6} w_{2}^{2} w_{1}+f_{7} w_{2} w_{1}^{2} \\
& +f_{8} w_{1}^{3}=k_{2} \cos \Omega_{0} \tau
\end{aligned}
$$

where $w_{1}$ and $w_{2}$ are the amplitudes of the first two modes, $\Phi$ are mode shape functions, $\gamma_{1}$ and $\gamma_{2}$ are modal damping ratios, and $\omega_{1}$ and $\omega_{2}$ are the natural frequencies of the first two modes. The expressions of the coefficients in (13a) and (13b) are given as follows:

$$
\begin{aligned}
\Phi & =\left[\begin{array}{ll}
\Phi_{11} & \Phi_{12} \\
\Phi_{21} & \Phi_{22}
\end{array}\right], \\
k_{1} & =\Phi_{11} P_{1}+\Phi_{21} P_{2} \\
k_{2} & =\Phi_{12} P_{1}+\Phi_{22} P_{2} \\
\omega_{1}^{2} & =\Phi_{11}^{2} B_{11}+\Phi_{11} \Phi_{21}\left(B_{12}+B_{21}\right)+\Phi_{21}^{2} B_{22} \\
\omega_{2}^{2} & =\Phi_{12}^{2} B_{11}+\Phi_{12} \Phi_{22}\left(B_{12}+B_{21}\right)+\Phi_{22}^{2} B_{22} \\
f_{1} & =\Phi_{11}^{3}\left(\Phi_{11} F_{q_{1}^{3}, 1}+\Phi_{21} F_{q_{1}^{3}, 2}\right) \\
& +\Phi_{11}^{2} \Phi_{21}\left(\Phi_{11} F_{q_{1}^{2} q_{2}, 1}+\Phi_{21} F_{q_{1}^{2} q_{2}, 2}\right) \\
& +\Phi_{11} \Phi_{21}^{2}\left(\Phi_{11} F_{q_{1} q_{2}^{2}, 1}+\Phi_{21} F_{q_{1} q_{2}^{2}, 2}\right) \\
& +\Phi_{21}^{3}\left(\Phi_{11} F_{q_{2}^{3}, 1}+\Phi_{21} F_{q_{2}^{3}, 2}\right) \\
f_{2} & =3 \Phi_{11}^{2} \Phi_{12}\left(\Phi_{11} F_{q_{1}^{3}, 1}+\Phi_{21} F_{q_{1}^{3}, 2}\right)
\end{aligned}
$$

$$
\begin{aligned}
& +\left(\Phi_{11}^{2} \Phi_{22}+2 \Phi_{11} \Phi_{12} \Phi_{21}\right) \\
& \cdot\left(\Phi_{11} F_{q_{1}^{2} q_{2}, 1}+\Phi_{21} F_{q_{1}^{2} q_{2}, 2}\right) \\
& +\left(\Phi_{12} \Phi_{21}^{2}+2 \Phi_{11} \Phi_{21} \Phi_{22}\right) \\
& \cdot\left(\Phi_{11} F_{q_{1} q_{2}^{2}, 1}+\Phi_{21} F_{q_{1} q_{2}^{2}, 2}\right) \\
& +3 \Phi_{21}^{2} \Phi_{22}\left(\Phi_{11} F_{q_{2}^{3}, 1}+\Phi_{21} F_{q_{2}^{3}, 2}\right) \\
& f_{3}=3 \Phi_{11} \Phi_{12}^{2}\left(\Phi_{11} F_{q_{1}^{3}, 1}+\Phi_{21} F_{q_{1}^{3}, 2}\right) \\
& +\left(\Phi_{12}^{2} \Phi_{21}+2 \Phi_{11} \Phi_{12} \Phi_{22}\right) \\
& \cdot\left(\Phi_{11} F_{q_{1}^{2} q_{2}, 1}+\Phi_{21} F_{q_{1}^{2} q_{2}, 2}\right) \\
& +\left(\Phi_{11} \Phi_{22}^{2}+2 \Phi_{12} \Phi_{21} \Phi_{22}\right) \\
& \cdot\left(\Phi_{11} F_{q_{1} q_{2}^{2}, 1}+\Phi_{21} F_{q_{1} q_{2}^{2}, 2}\right) \\
& +3 \Phi_{21} \Phi_{22}^{2}\left(\Phi_{11} F_{q_{2}^{3}, 1}+\Phi_{21} F_{q_{2}^{3}, 2}\right) \\
& f_{4}=\Phi_{12}^{3}\left(\Phi_{11} F_{q_{1}^{3}, 1}+\Phi_{21} F_{q_{1}^{3}, 2}\right) \\
& +\Phi_{12}^{2} \Phi_{22}\left(\Phi_{11} F_{q_{1}^{2} q_{2}, 1}+\Phi_{21} F_{q_{1}^{2} q_{2}, 2}\right) \\
& +\Phi_{12} \Phi_{22}^{2}\left(\Phi_{11} F_{q_{1} q_{2}^{2}, 1}+\Phi_{21} F_{q_{1} q_{2}^{2}, 2}\right) \\
& +\Phi_{22}^{3}\left(\Phi_{11} F_{q_{2}^{3}, 1}+\Phi_{21} F_{q_{2}^{3}, 2}\right) \\
& f_{5}=\Phi_{11}^{3}\left(\Phi_{12} F_{q_{1}^{3}, 1}+\Phi_{22} F_{q_{1}^{3}, 2}\right) \\
& +\Phi_{11}^{2} \Phi_{21}\left(\Phi_{12} F_{q_{1}^{2} q_{2}, 1}+\Phi_{22} F_{q_{1}^{2} q_{2}, 2}\right) \\
& +\Phi_{11} \Phi_{21}^{2}\left(\Phi_{12} F_{q_{1} q_{2}^{2}, 1}+\Phi_{22} F_{q_{1} q_{2}^{2}, 2}\right) \\
& +\Phi_{21}^{3}\left(\Phi_{12} F_{q_{2}^{3}, 1}+\Phi_{22} F_{q_{2}^{3}, 2}\right) \\
& f_{6}=3 \Phi_{11} \Phi_{12}^{2}\left(\Phi_{12} F_{q_{1}^{3}, 1}+\Phi_{22} F_{q_{1}^{3}, 2}\right) \\
& +\left(\Phi_{12}^{2} \Phi_{21}+2 \Phi_{11} \Phi_{12} \Phi_{22}\right) \\
& \cdot\left(\Phi_{12} F_{q_{1}^{2} q_{2}, 1}+\Phi_{22} F_{q_{1}^{2} q_{2}, 2}\right) \\
& +\left(\Phi_{11} \Phi_{22}^{2}+2 \Phi_{12} \Phi_{21} \Phi_{22}\right) \\
& \cdot\left(\Phi_{12} F_{q_{1} q_{2}^{2}, 1}+\Phi_{22} F_{q_{1} q_{2}^{2}, 2}\right) \\
& +3 \Phi_{21} \Phi_{22}^{2}\left(\Phi_{12} F_{q_{2}^{3}, 1}+\Phi_{22} F_{q_{2}^{3}, 2}\right) \\
& f_{7}=3 \Phi_{11}^{2} \Phi_{12}\left(\Phi_{12} F_{q_{1}^{3}, 1}+\Phi_{22} F_{q_{1}^{3}, 2}\right) \\
& +\left(\Phi_{11}^{2} \Phi_{22}+2 \Phi_{11} \Phi_{12} \Phi_{21}\right) \\
& \cdot\left(\Phi_{12} F_{q_{1}^{2} q_{2}, 1}+\Phi_{22} F_{q_{1}^{2} q_{2}, 2}\right) \\
& +\left(\Phi_{12} \Phi_{21}^{2}+2 \Phi_{11} \Phi_{21} \Phi_{22}\right)
\end{aligned}
$$




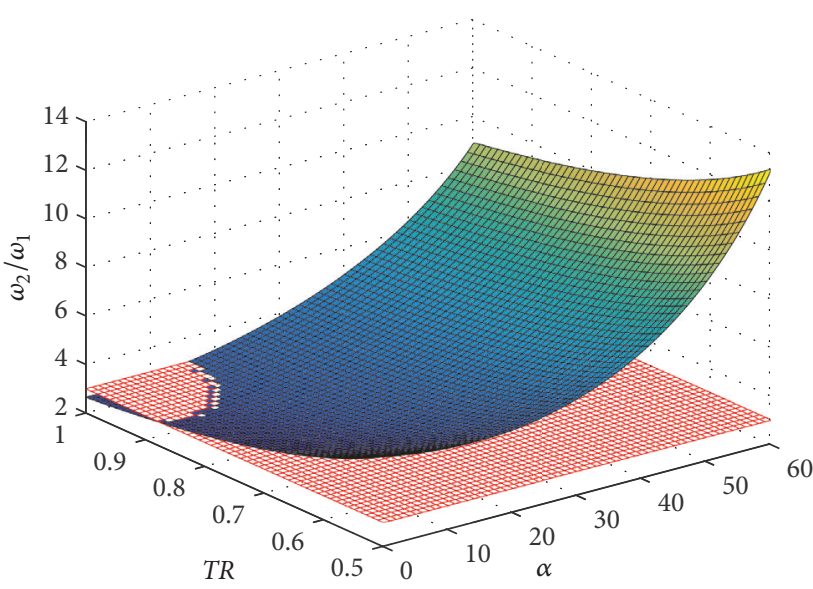

(a)

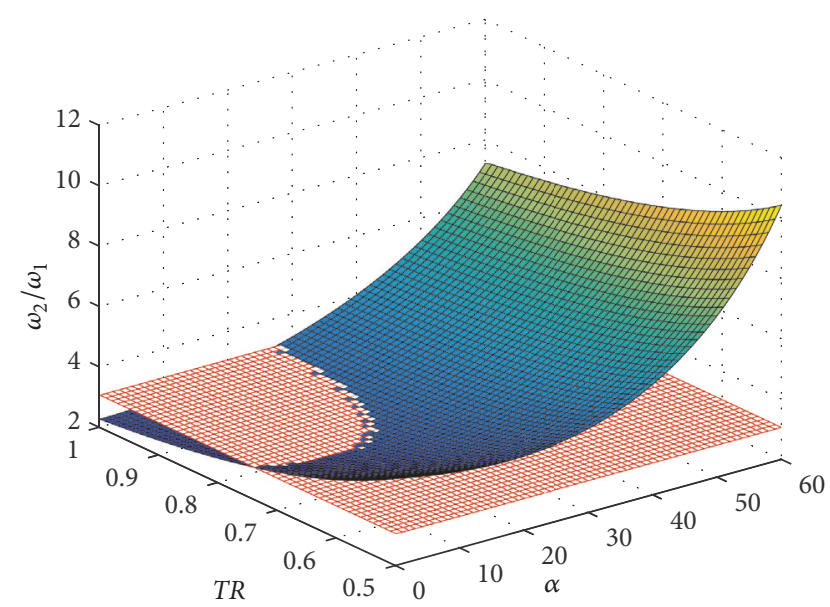

(b)

FIGURE 3: The variation of frequency ratio with different $T R$ and $\alpha$ for a trapezoidal cantilever plate with (a) $l=1$, (b) $l=0.8$.

$$
\begin{aligned}
& \cdot\left(\Phi_{12} F_{q_{1} q_{2}^{2}, 1}+\Phi_{22} F_{q_{1} q_{2}^{2}, 2}\right) \\
+ & 3 \Phi_{21}^{2} \Phi_{22}\left(\Phi_{12} F_{q_{2}^{3}, 1}+\Phi_{22} F_{q_{2}^{3}, 2}\right) \\
f_{8} & =\Phi_{11}^{3}\left(\Phi_{12} F_{q_{1}^{3}, 1}+\Phi_{22} F_{q_{1}^{3}, 2}\right) \\
+ & \Phi_{11}^{2} \Phi_{21}\left(\Phi_{12} F_{q_{1}^{2} q_{2}, 1}+\Phi_{22} F_{q_{1}^{2} q_{2}, 2}\right) \\
+ & \Phi_{11} \Phi_{21}^{2}\left(\Phi_{12} F_{q_{1} q_{2}^{2}, 1}+\Phi_{22} F_{q_{1} q_{2}^{2}, 2}\right) \\
& +\Phi_{21}^{3}\left(\Phi_{12} F_{q_{2}^{3}, 1}+\Phi_{22} F_{q_{2}^{3}, 2}\right)
\end{aligned}
$$

\section{Perturbation Analysis}

The condition of the 1:3 internal resonance could exist in the trapezoidal cantilever plate with the variation of geometrical parameters. As $c_{r}=1, h=0.01$ are fixed, the resonance condition can be satisfied by changing $T R, \alpha$, and $l$. Figures 2(a) and 2(b) show the variation of frequency ratio with different $T R$ and $\alpha$ for $l=1$ and $l=0.8$, respectively. As shown in Figure 3, several combinations of the parameters $T R, \alpha$, and $l$ can satisfy the condition of 1:3 internal resonance.

The multiple scales method is used to obtain the averaged equation of (13a) and (13b) for nonlinear dynamic analysis. Introducing time scales,

$$
T_{n}=\varepsilon^{n} t \quad(n=0,1,2, \cdots)
$$

where $\varepsilon$ is a small perturbation parameter and $m$ is the highest order of small parameter. The form of the asymptotic solution can be expressed as

$$
x(t, \varepsilon)=\sum_{n=0} \varepsilon^{n} x_{n}\left(T_{0}, T_{1}, T_{2}, \cdots, T_{m}\right)
$$

The derivatives with respect to $t$ are transformed into

$$
\frac{d}{d t}=\frac{\partial}{\partial T_{0}} \frac{d T_{0}}{d t}+\frac{\partial}{\partial T_{1}} \frac{d T_{1}}{d t}+\frac{\partial}{\partial T_{2}} \frac{d T_{2}}{d t}+\cdots
$$

$$
\begin{gathered}
+\frac{\partial}{\partial T_{m}} \frac{d T_{m}}{d t} \\
=D_{0}+\varepsilon D_{1}+\varepsilon^{2} D_{2}+\cdots+\varepsilon^{m} D_{m} \\
\frac{d^{2}}{d t^{2}}=\left(D_{0}+\varepsilon D_{1}+\varepsilon^{2} D_{2}+\cdots+\varepsilon^{m} D_{m}\right)^{2} \\
=D_{0}^{2}+2 \varepsilon D_{0} D_{1}+\varepsilon^{2}\left(D_{1}^{2}+2 D_{0} D_{2}\right)+\cdots
\end{gathered}
$$

where $D_{m}=\partial / \partial T_{m}$ and $m$ is determined by the requirement of solution precision. Considering the first-order precision solution with $O(\varepsilon)$, two time scales of $T_{0}$ and $T_{1}$ are adopted in the following analysis.

Here, the case of 1:3 internal resonance of the trapezoidal cantilever plate is considered. In the resonance, there exists

$$
\begin{aligned}
\omega_{1} & \approx \frac{1}{3} \omega_{2} \approx \frac{1}{3} \Omega_{0}, \\
3 \omega_{1} & =\Omega_{0}-\varepsilon \sigma_{1}, \\
\omega_{2} & =\Omega_{0}-\varepsilon \sigma_{2}
\end{aligned}
$$

where $\omega_{1}$ and $\omega_{2}$ are the first two natural frequencies of the induced linear system and $\sigma_{1}$ and $\sigma_{2}$ are two detuning parameters.

To obtain the averaged equation of (16) by using the multiple scales method, we specify the damping term, nonlinear term, and external excitation term as perturbations. Equations (13a) and (13b) are rewritten as

$$
\begin{aligned}
\ddot{w}_{1} & +\omega_{1}^{2} w_{1}=\varepsilon\left(k_{1} \cos \Omega_{0} \tau-\gamma_{1} \dot{w}_{1}-f_{1} w_{1}^{3}\right. \\
& \left.-f_{2} w_{1}^{2} w_{2}-f_{3} w_{1} w_{2}^{2}-f_{4} w_{2}^{3}\right) \\
\ddot{w}_{2} & +\omega_{2}^{2} w_{2}=\varepsilon\left(k_{2} \cos \Omega_{0} \tau-\gamma_{2} \dot{w}_{2}-f_{5} w_{2}^{3}\right. \\
& \left.-f_{6} w_{2}^{2} w_{1}-f_{7} w_{2} w_{1}^{2}-f_{8} w_{1}^{3}\right)
\end{aligned}
$$


The asymptotic solutions of (19a) and (19b) for $w_{1}(\tau, \varepsilon)$ and $w_{2}(\tau, \varepsilon)$ are

$$
\begin{aligned}
& w_{1}(\tau, \varepsilon)=w_{10}\left(T_{0}, T_{1}\right)+\varepsilon w_{11}\left(T_{0}, T_{1}\right) \\
& w_{2}(\tau, \varepsilon)=w_{20}\left(T_{0}, T_{1}\right)+\varepsilon w_{21}\left(T_{0}, T_{1}\right)
\end{aligned}
$$

Substituting (20a) and (20b) into (19a) and (19b) and equating the coefficients of similar power of $\varepsilon$, we can obtain

$\varepsilon^{0}:$

$$
\begin{aligned}
& D_{0}^{2} w_{10}+w_{10}=0 \\
& D_{0}^{2} w_{20}+w_{20}=0
\end{aligned}
$$

$$
\varepsilon^{1}:
$$

$$
\begin{aligned}
& D_{0}^{2} w_{11}+\frac{1}{9} \Omega_{0}^{2} w_{11} \\
& =-2 D_{0} D_{1} w_{10}-\gamma_{1} D_{0} w_{10}+2 \sigma_{1} w_{10}-f_{1} w_{10}^{3} \\
& \quad-f_{2} w_{10}^{2} w_{20}-f_{3} w_{10} w_{20}^{2}-f_{4} w_{20}^{3} \\
& \quad+k_{1} \cos \left(\Omega_{0} T_{0}\right) \\
& D_{0}^{2} w_{21}+\Omega_{0}^{2} w_{21} \\
& =-2 D_{0} D_{1} w_{20}-\gamma_{2} D_{0} w_{20}+2 \sigma_{2} w_{20}-f_{5} w_{20}^{3} \\
& \quad-f_{6} w_{20}^{2} w_{10}-f_{7} w_{20} w_{10}^{2}-f_{8} w_{10}^{3} \\
& \quad+k_{2} \cos \left(\Omega_{0} T_{0}\right)
\end{aligned}
$$

The general solution of (21a) can be written as

$$
\begin{aligned}
& w_{10}=\mathrm{A}_{1}\left(T_{1}\right) e^{(1 / 3) i \Omega_{0} T_{0}}+\overline{\mathrm{A}}_{1}\left(T_{1}\right) e^{-(1 / 3) i \Omega_{0} T_{0}} \\
& w_{20}=\mathrm{A}_{2}\left(T_{1}\right) e^{i \Omega_{0} T_{0}}+\overline{\mathrm{A}}_{2}\left(T_{1}\right) e^{-i \Omega_{0} T_{0}}
\end{aligned}
$$

where $A_{1}$ and $A_{2}$ are complex functions in $T_{1}$.

Substituting (22a) and (22b) into (21b), we obtain the following equation:

$$
\begin{aligned}
& D_{0}^{2} w_{11}+\frac{1}{9} \Omega_{0}^{2} w_{11}=\left(-\frac{2}{3} i \Omega_{0} D_{1} \mathrm{~A}_{1}-\frac{1}{3} i \Omega_{0} \gamma_{1} \mathrm{~A}_{1}\right. \\
& \left.+2 \sigma_{1} \mathrm{~A}_{1}-3 f_{1} \mathrm{~A}_{1}^{2} \overline{\mathrm{A}}_{1}-f_{2} \overline{\mathrm{A}}_{1}^{2} \mathrm{~A}_{2}-2 f_{3} \mathrm{~A}_{1} \mathrm{~A}_{2} \overline{\mathrm{A}}_{2}\right) \\
& \cdot e^{(1 / 3) i \Omega_{0} T_{0}}+c c+\mathrm{NST} \\
& D_{0}^{2} w_{21}+\Omega_{0}^{2} w_{21}=\left(-2 i \Omega_{0} D_{1} \mathrm{~A}_{2}-i \Omega_{0} \gamma_{2} \mathrm{~A}_{2}+2 \sigma_{2} \mathrm{~A}_{2}\right. \\
& \left.-3 f_{5} \mathrm{~A}_{2}^{2} \overline{\mathrm{A}}_{2}-2 f_{7} \mathrm{~A}_{1} \overline{\mathrm{A}}_{1} \mathrm{~A}_{2}-f_{8} \mathrm{~A}_{1}^{3}+\frac{1}{2} k_{2}\right) e^{i \Omega_{0} T_{0}} \\
& +c c+\mathrm{NST}
\end{aligned}
$$

where $c c$ stands for the complex conjugate of the former terms and NST stands for the terms that do not produce secular terms.

In order to facilitate the following analysis, set $\Omega_{0}=$ 1. Eliminating the secular terms from (23a) and (23b), the following averaged equations can be obtained:

$$
\begin{aligned}
D_{1} \mathrm{~A}_{1}= & -\frac{1}{2} \gamma_{1} \mathrm{~A}_{1}-3 i \sigma_{1} \mathrm{~A}_{1}+\frac{9 i}{2} f_{1} \mathrm{~A}_{1}^{2} \overline{\mathrm{A}}_{1} \\
& +\frac{3 i}{2} f_{2} \overline{\mathrm{A}}_{1}^{2} \mathrm{~A}_{2}+3 i f_{3} \mathrm{~A}_{1} \mathrm{~A}_{2} \overline{\mathrm{A}}_{2} \\
D_{1} \mathrm{~A}_{2}= & -\frac{1}{2} \gamma_{2} \mathrm{~A}_{2}-i \sigma_{2} \mathrm{~A}_{2}+\frac{3 i}{2} f_{5} \mathrm{~A}_{2}^{2} \overline{\mathrm{A}}_{2}+i f_{7} \mathrm{~A}_{1} \overline{\mathrm{A}}_{1} \mathrm{~A}_{2} \\
& +\frac{i}{2} f_{8} \mathrm{~A}_{1}^{3}-\frac{i}{4} k_{2}
\end{aligned}
$$

The functions $A_{1}$ and $A_{2}$ are expressed in the polar form:

$$
\begin{aligned}
& \mathrm{A}_{1}=\frac{1}{2} a_{1} e^{i \beta_{1}}, \\
& \mathrm{~A}_{2}=\frac{1}{2} a_{2} e^{i \beta_{2}}
\end{aligned}
$$

where $a_{i}$ and $\beta_{i}(i=1,2)$ are the functions with respect to the amplitude and phase of motion.

Substituting (25) into (24a) and (24b) and separating the real and imaginary parts, the averaged equations in the polar form are given as follows:

$$
\begin{aligned}
\dot{a}_{1}= & -\frac{1}{2} \gamma_{1} a_{1}-\frac{3}{8} f_{2} a_{1}^{2} a_{2} \sin \left(\beta_{2}-3 \beta_{1}\right) \\
a_{1} \dot{\beta}_{1}= & -3 \sigma_{1} a_{1}+\frac{9}{8} f_{1} a_{1}^{3}+\frac{3}{8} f_{2} a_{1}^{2} a_{2} \cos \left(\beta_{2}-3 \beta_{1}\right) \\
& +\frac{3}{4} f_{3} a_{1} a_{2}^{2} \\
\dot{a}_{2}= & -\frac{1}{2} \gamma_{2} a_{2}-\frac{1}{8} f_{8} a_{1}^{3} \sin \left(3 \beta_{1}-\beta_{2}\right)-\frac{1}{2} k_{2} \sin \beta_{2} \\
a_{2} \dot{\beta}_{2}= & -\sigma_{2} a_{2}+\frac{3}{8} f_{5} a_{2}^{3}+\frac{1}{4} f_{7} a_{1}^{2} a_{2} \\
& +\frac{1}{8} f_{8} a_{1}^{3} \cos \left(3 \beta_{1}-\beta_{2}\right)-\frac{1}{2} k_{2} \cos \beta_{2}
\end{aligned}
$$

\section{Analysis of Amplitude-Frequency Response}

For the periodic solutions of the system, $a_{1}, \beta_{1}, a_{2}$, and $\beta_{2}$ in the averaged equations are constants. Hence, set $\dot{a}_{1}=\dot{a}_{2}=$ 
$\dot{\beta}_{1}=\dot{\beta}_{2}=0$. Eliminating the terms of $\beta_{2}-3 \beta_{1}$, the amplitudefrequency response behaviors of the trapezoidal cantilever plate can be obtained

$$
\begin{aligned}
& \left(\frac{1}{2} \gamma_{1} a_{1}\right)^{2}+\left(3 \sigma_{1} a_{1}-\frac{9}{8} f_{1} a_{1}^{3}-\frac{3}{4} f_{3} a_{1} a_{2}^{2}\right)^{2} \\
& \quad=\left(\frac{3}{8} f_{2} a_{1}^{2} a_{2}\right)^{2} \\
& \left(\frac{1}{2} \gamma_{2} a_{2}+\frac{1}{2} k_{2} \sin \beta_{2}\right)^{2} \\
& \quad+\left(\sigma_{2} a_{2}-\frac{3}{8} f_{5} a_{2}^{3}-\frac{1}{4} f_{7} a_{1}^{2} a_{2}+\frac{1}{2} k_{2} \cos \beta_{2}\right)^{2} \\
& \quad=\left(\frac{1}{8} f_{8} a_{1}^{3}\right)^{2}
\end{aligned}
$$

According to the vibration amplitudes of the two modes, the steady-state vibration of the system can be divided into the following three conditions.

(a) When the vibration amplitude of one mode is zero and the amplitude of the other mode changes with the variation of excitation amplitude or frequency, these two modes are noncoupled.

(b) When the vibration amplitude of one mode is constant and the amplitude of the other mode changes with the variation of excitation amplitude or frequency, these two modes are weakly coupled.

(c) When the vibration amplitudes of the two modes both change with the variation of excitation amplitude or frequency, these two modes are strongly coupled.

Based on these three conditions, the nonlinear vibration behaviors of the trapezoidal cantilever plate are discussed as follows. In all cases, the structural parameters of the trapezoidal plate are chosen as $\alpha=23^{\circ}, T R=0.81$, and $l=0.8$, and the excitation point located at $\xi_{e}=0.25, \eta_{e}=0.75$. Thus, the natural frequencies of the corresponding induced linear system are $\omega_{1}=10.493 \mathrm{~Hz}$ and $\omega_{2}=31.492 \mathrm{~Hz}$, respectively.

4.1. Amplitude-Frequency Characteristics of Noncoupled Case. When the two modes are noncoupled, setting $a_{2}=0$ in (27a) and $a_{1}=0$ in (27b), then, (27a) and (27b) can be written as

$$
\begin{aligned}
& \left(\frac{1}{2} \gamma_{1} a_{1}\right)^{2}+\left(3 \sigma_{1} a_{1}-\frac{9}{8} f_{1} a_{1}^{3}\right)^{2}=0 \\
& \left(\frac{1}{2} \gamma_{2} a_{2}+\frac{1}{2} k_{2} \sin \beta_{2}\right)^{2} \\
& \quad+\left(\sigma_{2} a_{2}-\frac{3}{8} f_{5} a_{2}^{3}+\frac{1}{2} k_{2} \cos \beta_{2}\right)^{2}=0
\end{aligned}
$$

In order to satisfy (28a) and (28b), the following relations can be obtained:

$$
\begin{aligned}
& a_{1}=0 \\
& \sigma_{2}=\frac{3}{8} f_{5} a_{2}^{2} \pm \frac{\sqrt{k_{2}^{2}-\gamma_{2}^{2} a_{2}^{2}}}{2 a_{2}}
\end{aligned}
$$

\subsection{Amplitude-Frequency Characteristics of Weakly Coupled} Case. When the two modes are weakly coupled, setting $a_{2}$ as constant in (27a) and setting $a_{1}$ as constant in (27b), to simplify calculation, (27a) and (27b) can be written as

$$
\begin{aligned}
& \left(\frac{1}{2} \gamma_{1} a_{1}\right)^{2}+\left(3 \sigma_{1} a_{1}-\frac{9}{8} f_{1} a_{1}^{3}-\frac{3}{4} f_{3} a_{1}\right)^{2} \\
& \quad=\left(\frac{3}{8} f_{2} a_{1}^{2}\right)^{2} \\
& \left(\frac{1}{2} \gamma_{2} a_{2}+\frac{1}{2} k_{2} \sin \beta_{2}\right)^{2} \\
& \quad+\left(\sigma_{2} a_{2}-\frac{3}{8} f_{5} a_{2}^{3}-\frac{1}{4} f_{7} a_{2}+\frac{1}{2} k_{2} \cos \beta_{2}\right)^{2} \\
& =\left(\frac{1}{8} f_{8}\right)^{2}
\end{aligned}
$$

In order to satisfy (28a) and (28b), the following relations can be obtained

$$
\begin{aligned}
& \sigma_{1}=\frac{3}{8} f_{1} a_{1}^{2}+\frac{1}{4} f_{3} \pm \sqrt{\left(\frac{1}{8} f_{2} a_{1}\right)^{2}-\left(\frac{1}{6} \gamma_{1}\right)^{2}} \\
& \sigma_{2} \\
& =\frac{3 f_{5} a_{2}^{3}+2 f_{7} a_{2}-4 k_{2} \cos \beta_{2} \pm \sqrt{\left(f_{8}\right)^{2}-\left(4 \gamma_{2} a_{2}+4 k_{2} \sin \beta_{2}\right)^{2}}}{8 a_{2}}
\end{aligned}
$$

\subsection{Amplitude-Frequency Characteristics of Strongly Coupled} Case. For the stongly coupled case, the motion of the system is dominated by the two modes under a single frequency excitation. When excitation frequency has slight change, the amplitudes of the two modes are influenced to various extents. From (27a), the following equations can be obtained:

$$
\begin{aligned}
& a_{1}^{2}=\chi_{1}=\frac{\left(f_{2}^{2}-12 f_{1} f_{3}\right) a_{2}^{2}+48 f_{1} \sigma_{1} \pm \sqrt{\left(f_{2}^{4}-24 f_{1} f_{2}^{2} f_{3}\right) a_{2}^{4}+96 f_{1} f_{2}^{2} \sigma_{1} a_{2}^{2}-64 f_{1}^{2} \gamma_{1}^{2}}}{18 f_{1}^{2}} \\
& a_{2}^{2}=\chi_{2}=\frac{\left(3 f_{2}^{2}-36 f_{1} f_{3}\right) a_{1}^{2}+96 f_{3} \sigma_{1} \pm \sqrt{\left(9 f_{2}^{4}-216 f_{1} f_{2}^{2} f_{3}\right) a_{1}^{4}+576 f_{2}^{2} f_{3} \sigma_{1} a_{1}^{2}-256 f_{3}^{2} \gamma_{1}^{2}}}{24 f_{3}^{2}}
\end{aligned}
$$


Substituting (32a) and (32b) into (27b), respectively, the amplitude-frequency relations of the two modes can be obtained:

$$
\begin{aligned}
& \left(\frac{1}{2} \gamma_{2} \pm \frac{k_{2}}{2 \sqrt{\chi_{2}}} \sin \beta_{2}\right)^{2} \\
& +\left(\sigma_{2}-\frac{3}{8} f_{5} \chi_{2}-\frac{1}{4} f_{7} a_{1}^{2} \pm \frac{k_{2}}{2 \sqrt{\chi_{2}}} \cos \beta_{2}\right)^{2} \\
& \quad=\frac{f_{8}^{2} a_{1}^{6}}{64 \chi_{2}} \\
& \left(\frac{1}{2} \gamma_{2}+\frac{k_{2}}{2 a_{2}} \sin \beta_{2}\right)^{2} \\
& \quad+\left(\sigma_{2}-\frac{3}{8} f_{5} a_{2}^{2}-\frac{1}{4} f_{7} \chi_{1}+\frac{k_{2}}{2 a_{2}} \cos \beta_{2}\right)^{2}=\frac{f_{8}^{2} \chi_{1}^{3}}{64 a_{2}^{2}}
\end{aligned}
$$

\section{Results and Discussions}

In this section, the amplitude-frequency behaviors and nonlinear dynamic responses of the trapezoidal cantilever plate with 1:3 internal resonance are discussed. In all cases, the structural parameters of the trapezoidal plate are chosen as $\alpha=30^{\circ}, T R=0.81$, and $l=0.8$ from Figure $3(\mathrm{~b})$ and the excitation point located at $\xi_{e}=0.25, \eta_{e}=0.75$. Thus, the natural frequencies of the corresponding induced linear system are $\omega_{1}=10.493 \mathrm{~Hz}$ and $\omega_{2}=31.492 \mathrm{~Hz}$, respectively, which can satisfy the condition of 1:3 internal resonance.

\subsection{Amplitude-Frequency Characteristics}

5.1.1. Noncoupled Case. For the noncoupled case, it is seen from (29a) that when the excitation frequency approaches the frequency of the second mode, the motion of the first mode will not be excited. The amplitude-frequency curve of the second mode can be obtained from (29b). Figure 4 shows the amplitude-frequency curve of the second mode in the noncoupled case. Here, $\gamma_{1}=\gamma_{2}=0.01, P_{0}=0.01$. Its skeleton curve trends to the direction of frequency increment, which indicates a typical hardening-spring characteristic. The multivalue and jump phenomena exist for the second mode in the resonance region.

5.1.2. Weakly Coupled Case. For the weakly coupled case, the amplitude-frequency curves of these two modes are obtained from (31a) and (31b) shown in Figure 5, where $\gamma_{1}=\gamma_{2}=$ $0.01, P_{0}=0.01, \beta_{2}=\pi / 4$. Comparing with Figures 5(b) and 4 , the amplitude-frequency curve of the second mode in the noncoupled case can be obtained by shifting the plot of weakly coupled case to right. The results show that the amplitude-frequency curves exhibit hardening-spring behavior. It indicates that weakly coupled effect cannot change the nonlinear stiffness behavior but has a certain influence on resonance frequency of the system. An increscent resonance frequency in Figure 5(b) is shown in the present case. The multivalue phenomenon also exists for the second mode in

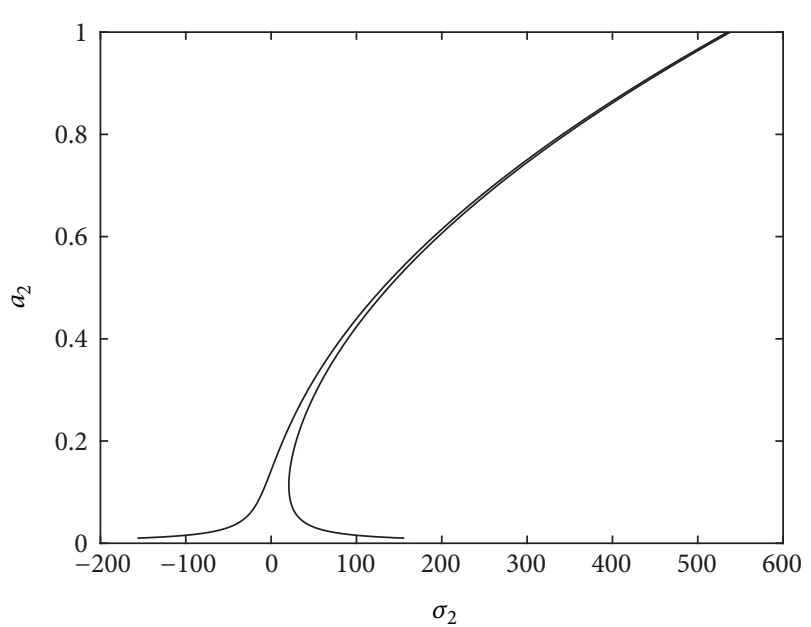

FIgURE 4: The amplitude-frequency curve of the second mode in the noncoupled case.

the resonance region, which can be clarified by the stability analysis of steady-state solutions. According to (26), setting $\sigma_{2}=100$, the phase plot of the second mode can be obtained, which is shown in Figure 6. It is found that the three steadystate solutions $S_{1}, S_{2}$, and $S_{3}$ correspond to the points of upper, middle, and lower solution branches at $\sigma_{2}=100$ in Figure 5(b), respectively. The phase trajectory in Figure 6 demonstrates that $S_{1}\left(a_{2}=0.04\right)$ and $S_{3}\left(a_{2}=0.2904\right)$ are stable focus points and $S_{2}\left(a_{2}=0.2503\right)$ is an unstable saddle point. There exists a line across the saddle point $S_{2}$, and the phase-plane is divided into two attraction basins $S_{1}$ and $S_{3}$. Therefore, the present system has two stable solutions and one unstable solution in the multivalue frequency band, but unstable motions can be only obtained in analytical solutions.

Based on the existence condition of steady-state solution, $\dot{\beta}_{2}=0$ and $\beta_{2} \in[-\pi, \pi]$. The effect of phase $\beta_{2}$ on the amplitude-frequency behavior of the second mode is analyzed. As for the small amplitude excitation, $\beta_{2}$ hardly affects the amplitude-frequency behavior. As for the large amplitude excitation, $\beta_{2}$ can change the shape of amplitudefrequency curve of the second mode but cannot affect the soft/hardening-spring characteristics of the mode. Figure 7 shows the amplitude-frequency curves of the second mode with different $\beta_{2}$ under $P_{0}=2$. The results demonstrate that with the increase of excitation amplitude, the effect of phase $\beta_{2}$ on the amplitude-frequency behavior becomes remarkable.

5.1.3. Strongly Coupled Case. According to the resonance relation $\sigma_{2}-\sigma_{1}=\left(3 \omega_{1}-\omega_{2}\right) / \varepsilon$ from (18), the difference of $\sigma_{1}$ and $\sigma_{2}$ is a constant. Substituting $\sigma_{2}=\sigma_{1}+$ constant into (33a), the amplitude-frequency relation of the first mode can be obtained. Similarly, the relation of the second mode can also be obtained from (33b). Figure 8 shows the amplitudefrequency curves of the system in the strongly coupled case. Here, $\sigma_{1}=\sigma_{2}, \gamma_{1}=\gamma_{2}=0.01, P_{0}=0.01, \beta_{2}=\pi / 4$. The results indicate that the first mode has hardening-spring behavior, 


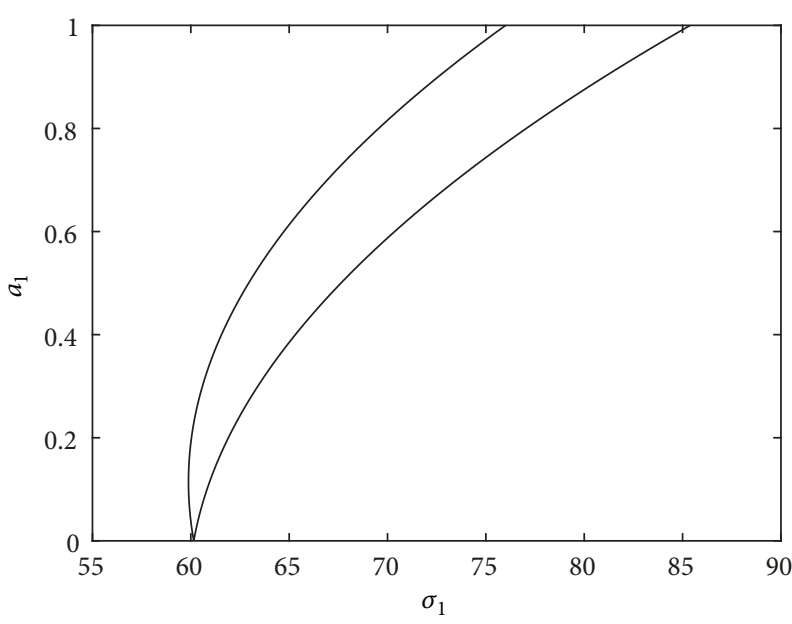

(a)

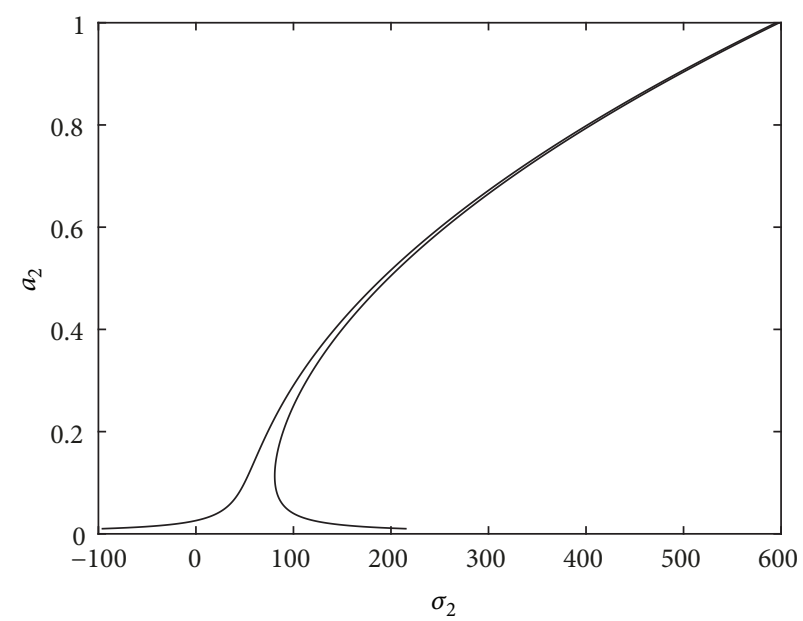

(b)

FIgURE 5: The amplitude-frequency curves of the two modes in the weakly coupled case.

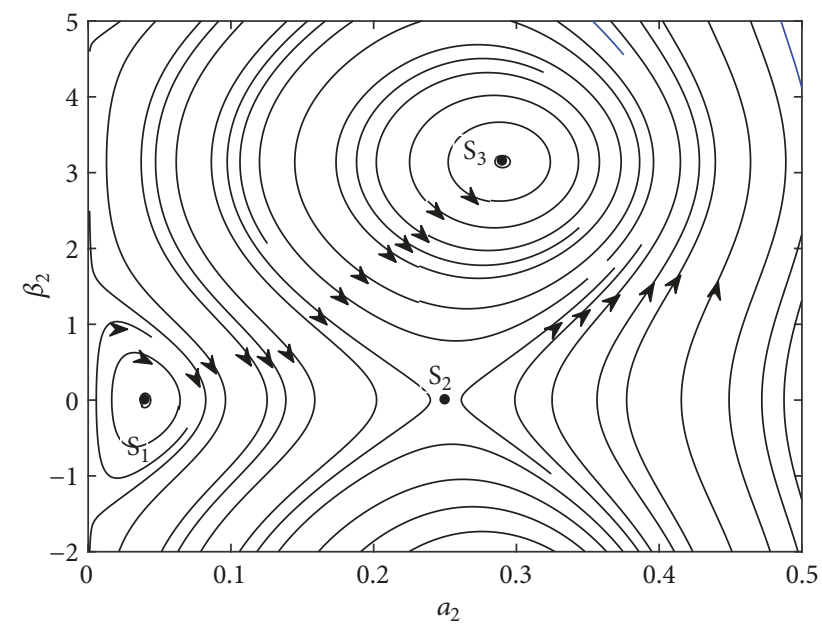

FIGURE 6: Phase plot of the second mode under the given detuning parameter $\left(\sigma_{2}=100\right)$.

but the shape of its amplitude-frequency curve is observably different from that of the weakly coupled case. However, the second mode becomes a soft-spring behavior with the leftward trend of its skeleton curve.

However, it is difficult to reach the accurate multiples of frequencies of the two modes for a practice model. The cases for $\sigma_{1} \neq \sigma_{2}$ are discussed in Figure 9. When $\sigma_{2}-\sigma_{1}>0$, the second frequency is slightly more than three times as large as the first frequency. When $\sigma_{2}-\sigma_{1}<0$, the second frequency is slightly less than three times as large as the first frequency. As shown in Figure 9, the values of $\sigma_{2}-\sigma_{1}$ have little effect on the amplitude-frequency behavior of the first mode. The amplitude-frequency curve of the second mode moves to the right and the corresponding peak increases with the increase of $\sigma_{2}-\sigma_{1}$. The results indicate that the rise of the difference of detuning parameters can increase the resonance frequency. Figure 10 shows the amplitude-frequency curves of the two modes for $\sigma_{2}-\sigma_{1}=10$ under different excitation amplitudes. The results show that the soft/hardening-spring characteristic of each mode cannot be affected by changing excitation amplitude. The local peak for the first mode slightly increases with the increase of excitation amplitude. On the contrary, the amplitude peak for the second mode decreases and the corresponding resonance frequency increases.

The case for $\sigma_{2}-\sigma_{1}=-5$ is shown in Figure 11. It can be seen that the first mode keeps a hardening-spring behavior, and the amplitude-frequency curve of the second mode exhibits a rightward trend, which shows hardening-spring behavior. Furthermore, comparing with the first mode, the effect of amplitude $P_{0}$ on the second mode is more significant, which shows that the vibration amplitude increases with the increasing of $P_{0}$.

5.2. Nonlinear Dynamic Responses. In order to analyze the nonlinear dynamic responses of the system, the averaged equations can be obtained by transforming (24a) and (24b) into the Cartesian form. The functions $A_{1}$ and $A_{2}$ can be expressed as

$$
\begin{aligned}
& \mathrm{A}_{1}=x_{1}+i x_{2}, \\
& \mathrm{~A}_{2}=x_{3}+i x_{4}
\end{aligned}
$$

where $x_{1}, x_{2}, x_{3}$, and $x_{4}$ are the functions with respect to the amplitude and phase of motions. Substituting (34) into (24a) and (24b), the averaged equations in the Cartesian form are obtained as follows:

$$
\begin{aligned}
\dot{x}_{1}= & -\frac{1}{2} \gamma_{1} x_{1}+3 \sigma_{1} x_{2}-\frac{9}{2} f_{1}\left(x_{1}^{2}+x_{2}^{2}\right) x_{2} \\
& -\frac{3}{2} f_{2}\left(x_{1}^{2}-x_{2}^{2}\right) x_{4}+3 f_{2} x_{1} x_{2} x_{3} \\
& -3 f_{3}\left(x_{3}^{2}+x_{4}^{2}\right) x_{2} \\
\dot{x}_{2}= & -\frac{1}{2} \gamma_{1} x_{2}-3 \sigma_{1} x_{1}+\frac{9}{2} f_{1}\left(x_{1}^{2}+x_{2}^{2}\right) x_{1}
\end{aligned}
$$




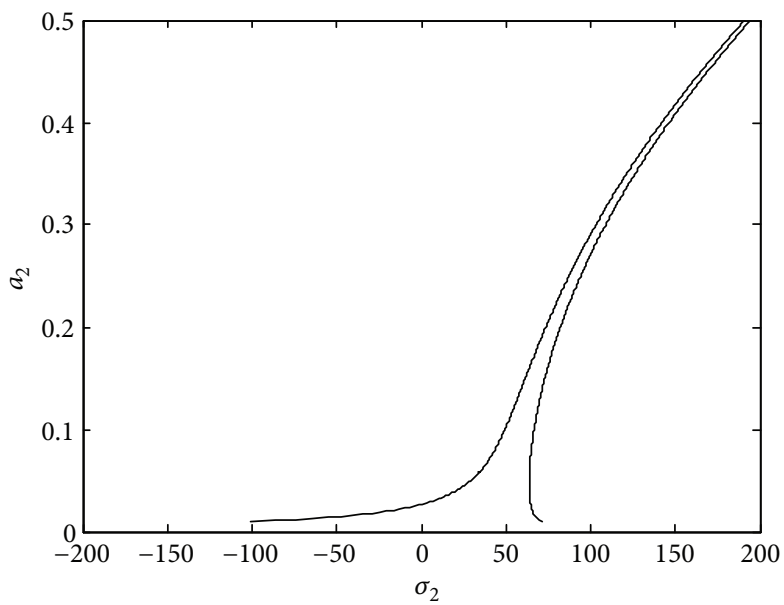

(a)

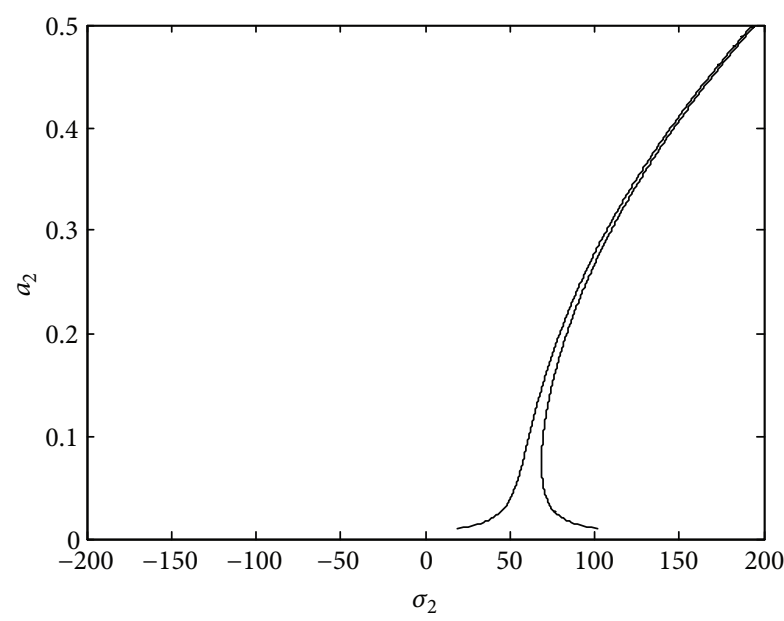

(c)

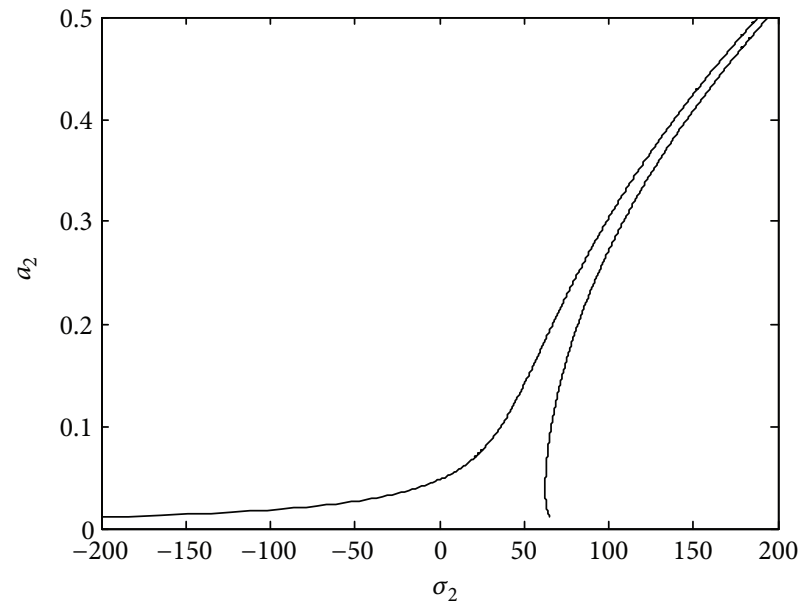

(b)

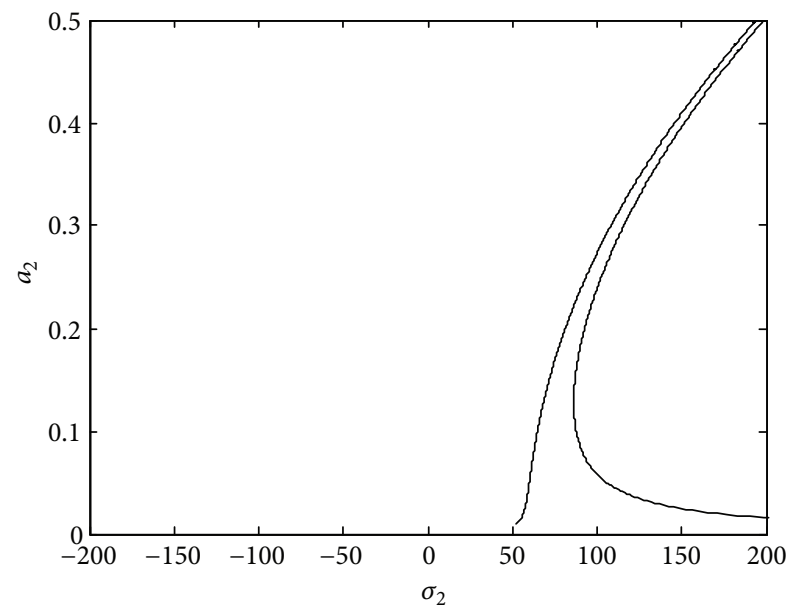

(d)

FiguRE 7: The amplitude-frequency curves of the second mode with different $\beta_{2}$ under $P_{0}=2$ : (a) $\beta_{2}=-1 / 3 \pi$; (b) $\beta_{2}=1 / 10 \pi$; (c) $\beta_{2}=1 / 2 \pi$; (d) $\beta_{2}=3 / 4 \pi$.

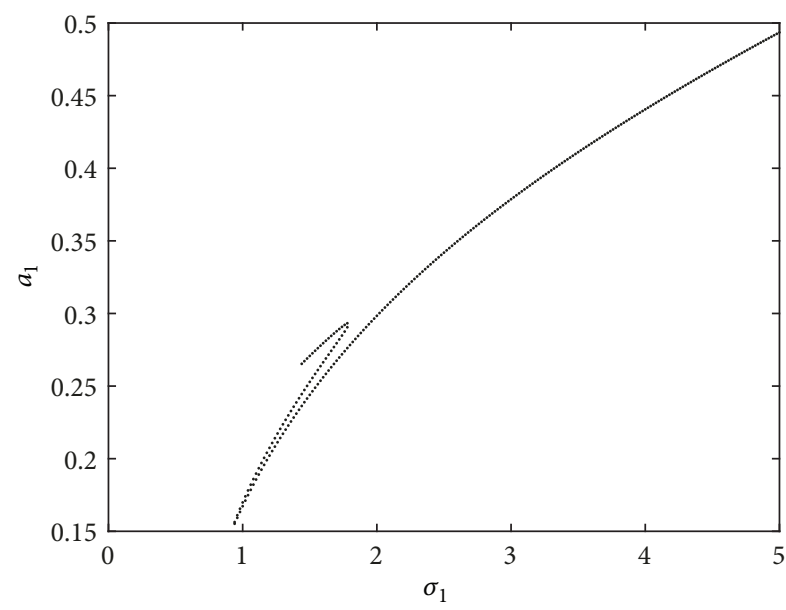

(a)

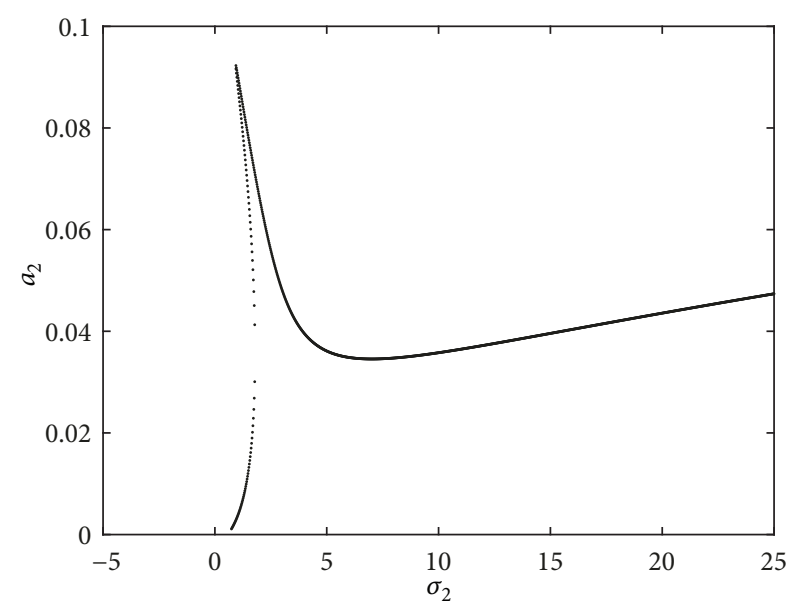

(b)

FIGURE 8: The amplitude-frequency curves of the system in the strongly coupled case. 


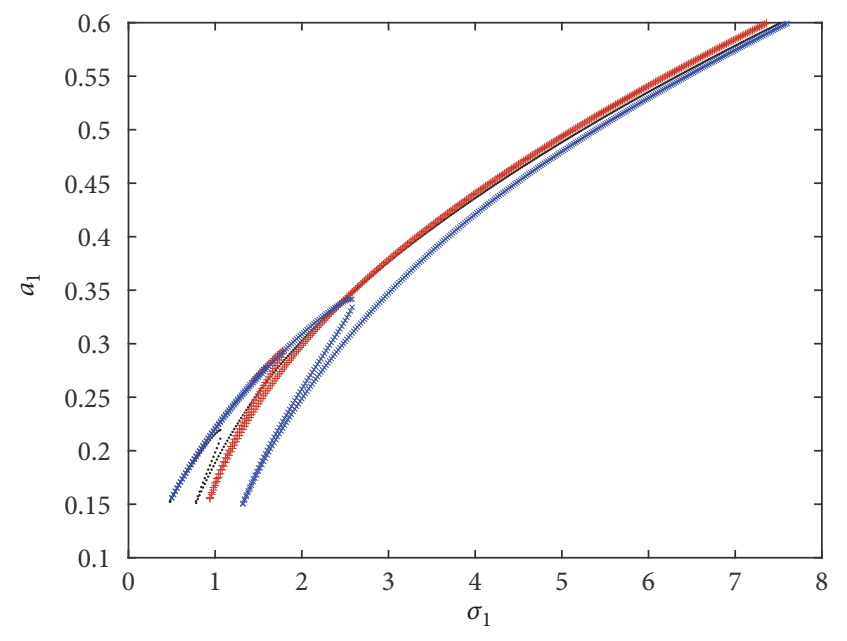

$$
\begin{aligned}
& \sigma_{2}-\sigma_{1}=-2 \\
& +\sigma_{2}-\sigma_{1}=0 \\
& \text { × } \sigma_{2}-\sigma_{1}=2
\end{aligned}
$$

(a)

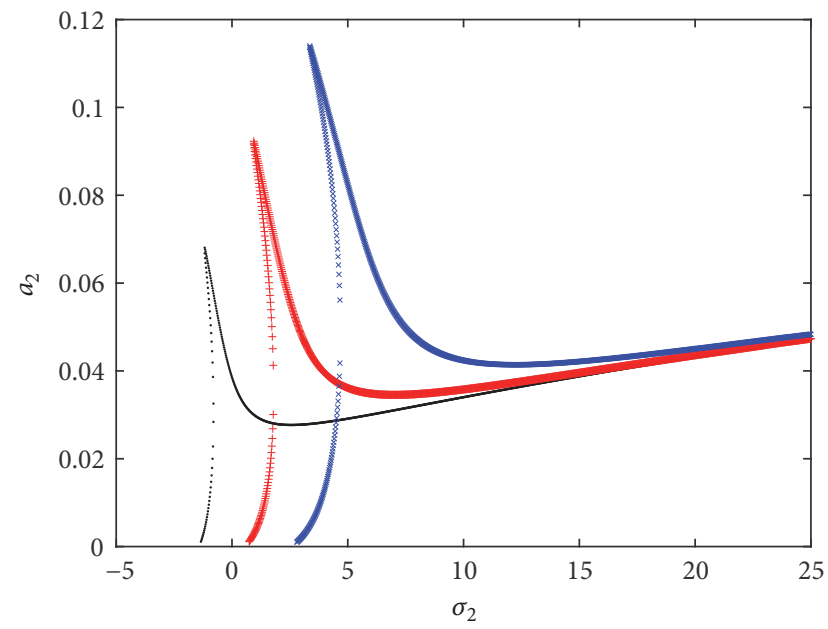

$\sigma_{2}-\sigma_{1}=-2$
$\sigma_{2}-\sigma_{1}=0$

$\times \sigma_{2}-\sigma_{1}=2$

Figure 9: The amplitude-frequency curves of the two modes for different values of $\sigma_{1}-\sigma_{2}$.
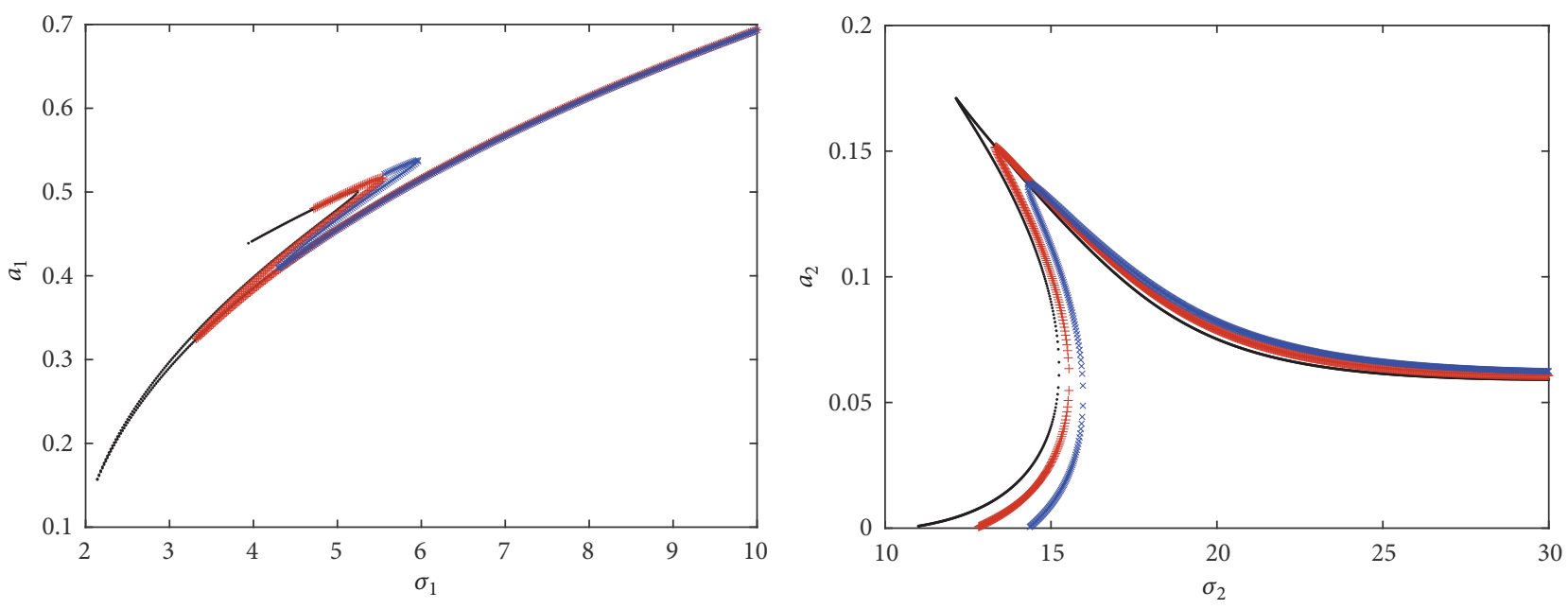

$$
\begin{aligned}
\cdot P_{0} & =0.01 \\
+P_{0} & =0.1 \\
\times P_{0} & =0.2
\end{aligned}
$$

$$
\begin{aligned}
\cdot P_{0} & =0.01 \\
+P_{0} & =0.1 \\
\times P_{0} & =0.2
\end{aligned}
$$

(a)

(b)

FIgURE 10: The amplitude-frequency curves of the two modes for $\sigma_{1}-\sigma_{2}=10$ under different excitation amplitude $P_{0}$.

$$
\begin{aligned}
& +\frac{3}{2} f_{2}\left(x_{1}^{2}-x_{2}^{2}\right) x_{3}+3 f_{2} x_{1} x_{2} x_{4} \\
& +3 f_{3}\left(x_{3}^{2}+x_{4}^{2}\right) x_{1} \\
\dot{x}_{3}= & -\frac{1}{2} \gamma_{2} x_{3}+\sigma_{2} x_{4}-\frac{3}{2} f_{5}\left(x_{3}^{2}+x_{4}^{2}\right) x_{4} \\
& -f_{7}\left(x_{1}^{2}+x_{2}^{2}\right) x_{4}-\frac{1}{2} f_{8}\left(3 x_{1}^{2} x_{2}-x_{2}^{3}\right) \\
\dot{x}_{4}= & -\frac{1}{2} \gamma_{2} x_{4}-\sigma_{2} x_{3}+\frac{3}{2} f_{5}\left(x_{3}^{2}+x_{4}^{2}\right) x_{3}
\end{aligned}
$$$$
+f_{7}\left(x_{1}^{2}+x_{2}^{2}\right) x_{3}+\frac{1}{2} f_{8}\left(x_{1}^{3}-3 x_{1} x_{2}^{2}\right)-\frac{1}{4} k_{2}
$$

The Jacobi matrix of (35) can be written as

$$
\mathbf{J}=\left[\begin{array}{llll}
g_{11} & g_{12} & g_{13} & g_{14} \\
g_{21} & g_{22} & g_{23} & g_{24} \\
g_{31} & g_{23} & g_{33} & g_{34} \\
g_{41} & g_{24} & g_{34} & g_{44}
\end{array}\right]
$$



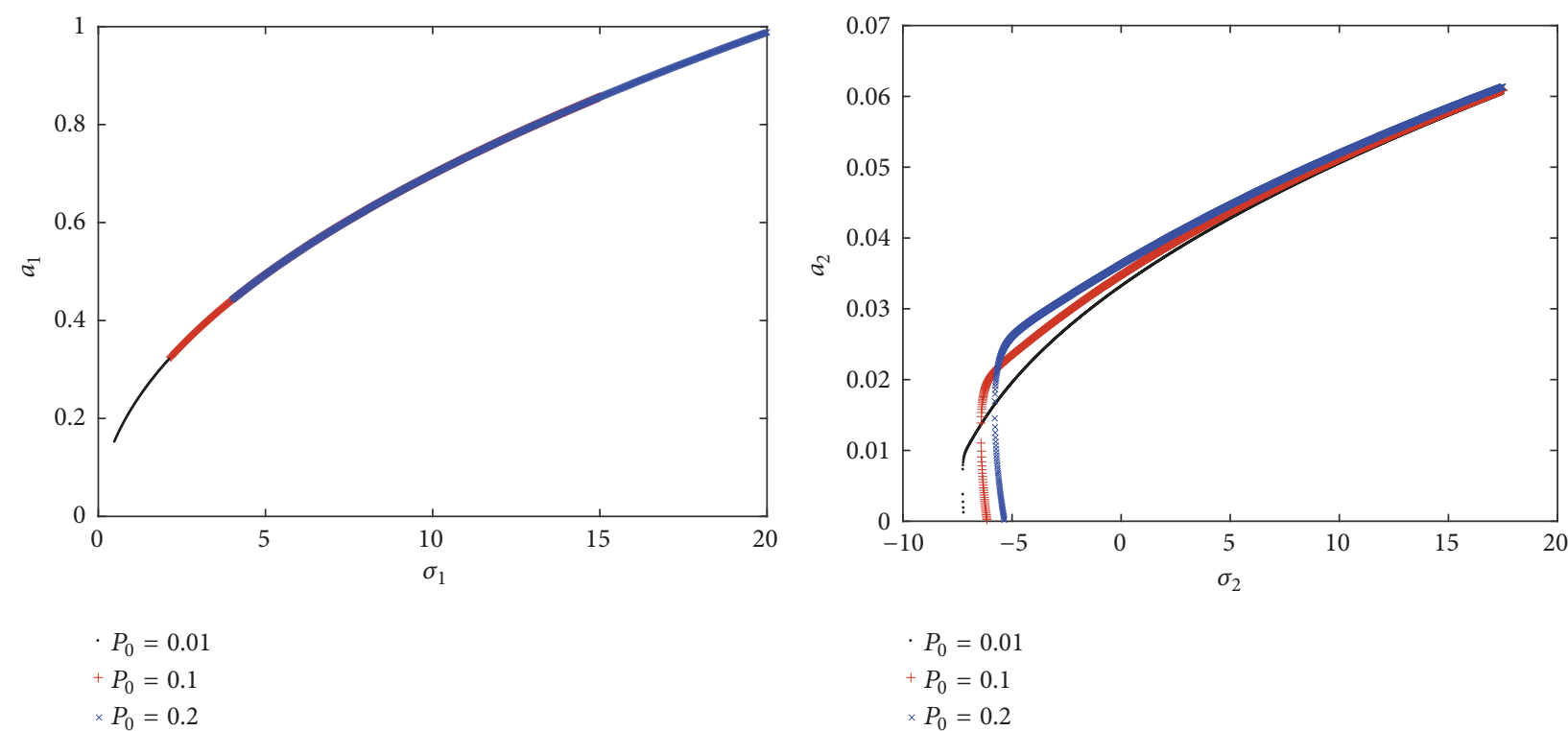

(a)

(b)

FIgURE 11: The amplitude-frequency curves of the two modes for $\sigma_{1}-\sigma_{2}=-5$ under different excitation amplitude $P_{0}$.

where

$$
\begin{aligned}
g_{11}= & -\frac{1}{2} \gamma_{1}-9 f_{1} x_{1} x_{2}-3 f_{2} x_{1} x_{4}+3 f_{2} x_{2} x_{3}, \\
g_{12}= & 3 \sigma_{1}-\frac{27}{2} f_{1} x_{2}^{2}+3 f_{2} x_{2} x_{4}+3 f_{2} x_{1} x_{3} \\
& -3 f_{3}\left(x_{3}^{2}+x_{4}^{2}\right), \\
g_{13}= & 3 f_{2} x_{1} x_{2}-6 f_{3} x_{2} x_{3}, \\
g_{14}= & -\frac{3}{2} f_{2}\left(x_{1}^{2}-x_{2}^{2}\right)-6 f_{3} x_{2} x_{4}, \\
g_{21}= & -3 \sigma_{1}+\frac{27}{2} f_{1} x_{1}^{2}+3 f_{2} x_{1} x_{3}+3 f_{2} x_{2} x_{4} \\
& +3 f_{3}\left(x_{3}^{2}+x_{4}^{2}\right), \\
g_{22}= & -\frac{1}{2} \gamma_{1}+9 f_{1} x_{1} x_{2}-3 f_{2} x_{2} x_{3}+3 f_{2} x_{1} x_{4}, \\
g_{23}= & \frac{3}{2} f_{2}\left(x_{1}^{2}-x_{2}^{2}\right)+6 f_{3} x_{1} x_{3}, \\
g_{34}= & \sigma_{2}-\frac{3}{2} f_{5}\left(x_{3}^{2}+3 x_{4}^{2}\right)-f_{7}\left(x_{1}^{2}+x_{2}^{2}\right), \\
g_{24}= & -\frac{1}{2} f_{2} x_{1} x_{2}+6 f_{3} x_{1} x_{4}, \\
g_{31}= & -2 f_{7} x_{1} x_{3}+\frac{3}{2} f_{8}\left(x_{3} x_{4},-x_{2}^{2}\right), \\
g_{32}= & -2 f_{7} x_{2} x_{4}-\frac{3}{2} f_{8}\left(x_{1}^{2}-x_{2}^{2}\right), \\
g_{33} & \\
g_{2} &
\end{aligned}
$$

$$
\begin{aligned}
& g_{42}=2 f_{7} x_{2} x_{3}-3 f_{8} x_{1} x_{2}, \\
& g_{43}=-\sigma_{2}+\frac{3}{2} f_{5}\left(3 x_{3}^{2}+x_{4}^{2}\right)+f_{7}\left(x_{1}^{2}+x_{2}^{2}\right), \\
& g_{44}=-\frac{1}{2} \gamma_{2}+3 f_{5} x_{3} x_{4} .
\end{aligned}
$$

The eigenvalue equation of the Jacobi matrix is

$$
D(\lambda)=|\lambda \mathbf{I}-\mathbf{J}|=0,
$$

and when the real parts of all eigenvalues are negative, the steady solutions are asymptotically stable.

The zero-solution stability of (35) can be determined; setting $x_{1}=x_{2}=x_{3}=x_{4}=0$, the eigenvalues of the Jacobi matrix are

$$
\begin{aligned}
& \lambda_{1,2}=-\frac{1}{2} \gamma_{1} \pm 3 \sigma_{1} \\
& \lambda_{3,4}=-\frac{1}{2} \gamma_{2} \pm \sigma_{2}
\end{aligned}
$$

Hence, the zero-solution stability of the system with 1:3 internal resonance depends on the modal damping ratios $\gamma_{1}, \gamma_{2}$. When the structure has positive damping, the zero solutions are stable. Otherwise, the zero solutions are unstable.

The averaged equation from (35) can be solved by numerical method [37] to analyze the nonlinear dynamic responses of trapezoidal cantilever plate subjected to transverse harmonic excitation. The excitation amplitude $P_{0}$ is selected as a controlling parameter to explore the influence of excitation amplitude on the system. Here, the system parameters and initial conditions are given as $\gamma_{1}=\gamma_{2}=0.01, \sigma_{1}=2, \sigma_{2}=$ $5, x_{10}=1.8, x_{20}=1.2, x_{30}=-0.5, x_{40}=1.4$. Figure 12 shows 


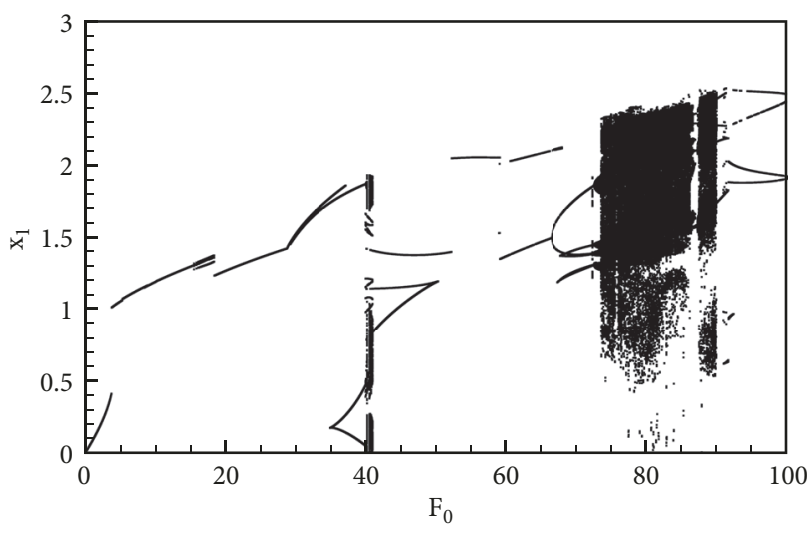

(a)

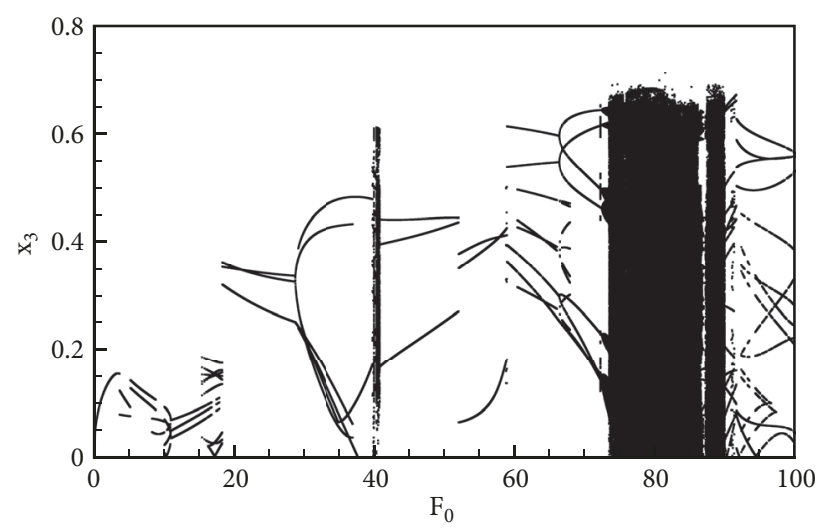

(b)

FIGURE 12: Bifurcation of the two modes for the trapezoidal plate.

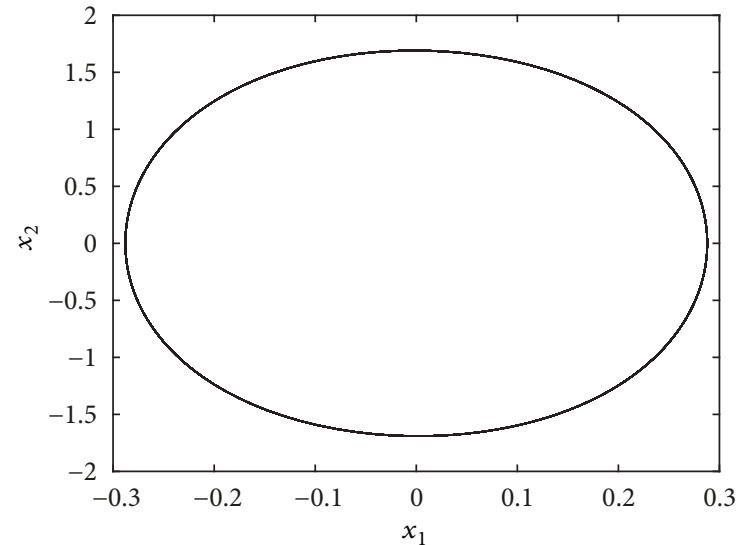

(a)

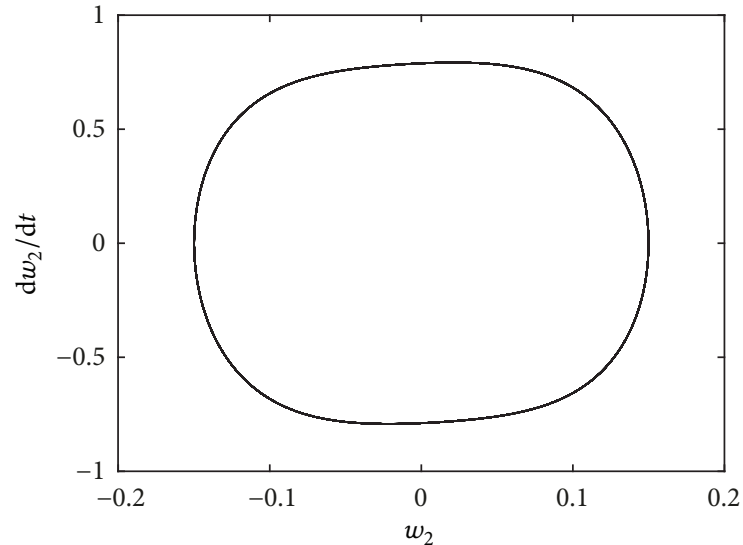

(b)

FIGURE 13: Period-1 motions of the two modes for the trapezoidal plate under excitation amplitude $P_{0}=3 \mathrm{~N}$.

the bifurcations of the two modes for the trapezoidal plate. Here, the modal damping ratios are $\gamma_{1}=\gamma_{2}=0.05$. The results indicate that the two modes both present a single periodic motion with small excitation amplitude, and with the increase of excitation amplitude, the nonlinear effect becomes more remarkable, which causes the occurrence of bifurcation. Later, with the further increase of $P_{0}$, the two modes of the system show multiperiod motion, quasi-period, and chaotic motions. Figure 13 shows the motions of the two modes at $P_{0}=3 \mathrm{~N}$. The two modes present period-1 motions. Figures $14-17$ give the motions of the two modes of the trapezoidal plate at $P_{0}=36 \mathrm{~N}, 40 \mathrm{~N}, 40.1 \mathrm{~N}, 40.5 \mathrm{~N}$, respectively. The results show that the first mode presents period-2, period-4, period-8, and chaotic motion, but the second mode presents period-3, period-6, period-12, and chaotic motion. It indicates that the route to chaos is via period-doubling for the present trapezoidal plate. Figures 18 and 19 show the motions of the two modes at $P_{0}=73 \mathrm{~N}$ and $P_{0}=80 \mathrm{~N}$, respectively. The Poincare maps from Figures 18(c) and 18 (d) show closed loops, which indicate a quasi-periodic motion. When the excitation amplitude $P_{0}$ increases to $80 \mathrm{~N}$, the Poincare maps in Figures 19(c) and 19(d) exhibit a cloud of points, which indicates that the response of the system turns to chaos.

The above numerical results demonstrate that when the system is subjected to the excitation with single frequency, the motion with multifrequency components is excited with the increase of excitation amplitude, which will exhibit complex nonlinear dynamic phenomena. Moreover, it is also found from the phase plots $\left(x_{1}, x_{2}\right),\left(x_{3}, x_{4}\right)$ that the amplitude of the first mode is larger than that of the second mode, which are in agreement with the amplitude-frequency results in Section 4. These results indicate the occurrence of internal resonance as a result of the coupling of the two modes.

The effect of modal damping ratio on nonlinear dynamic behaviors is also analyzed. Figure 20 shows the bifurcations of the two modes for the trapezoidal plate with $\gamma_{1}=\gamma_{2}=$ 0.1 . The chaos region of the system is reduced and both of the two modes show multiperiod motions. The modal damping ratio is further increased to $\gamma_{1}=\gamma_{2}=0.2$; the corresponding bifurcation diagrams are shown in Figure 21. It is found that the first mode always presents a single periodic motion. But the second presents single periodic motion with $P_{0}<4.7 \mathrm{~N}$ and becomes a period-3 motion with 


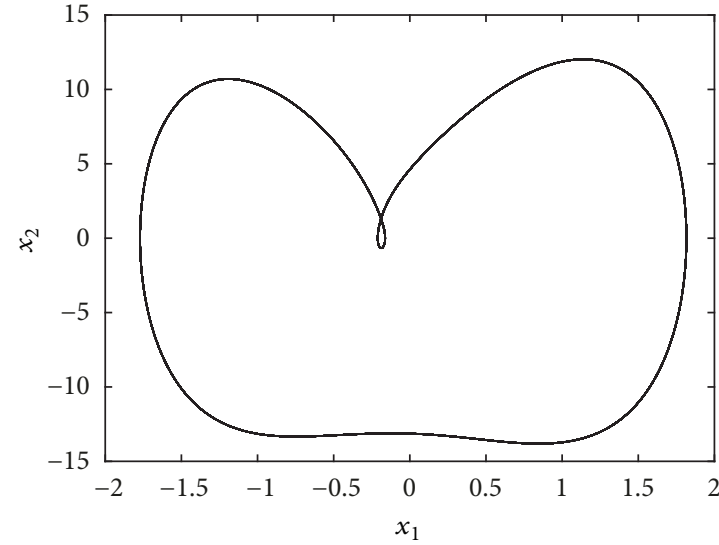

(a)

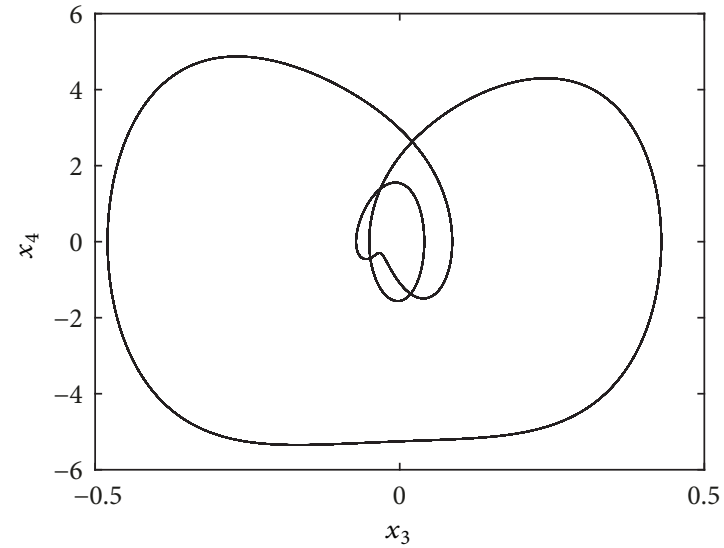

(b)

Figure 14: Multiperiod motions of the two modes for the trapezoidal plate under excitation amplitude $P_{0}=36 \mathrm{~N}$.

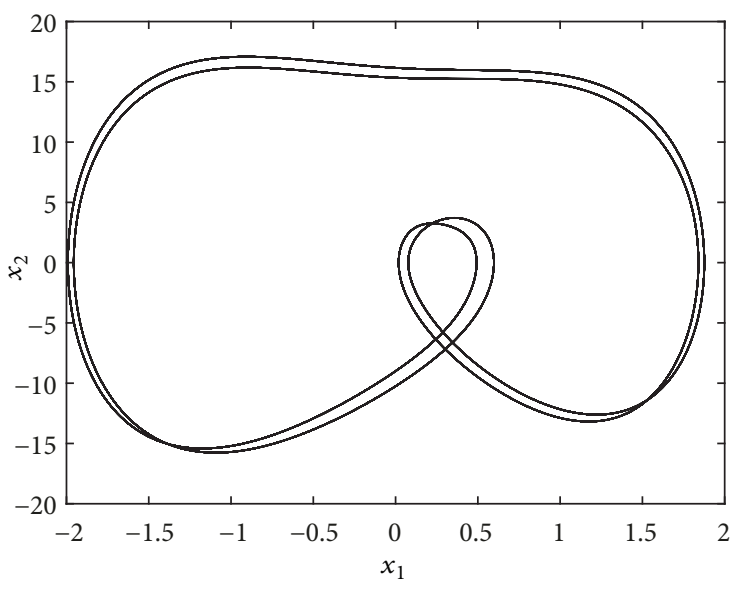

(a)

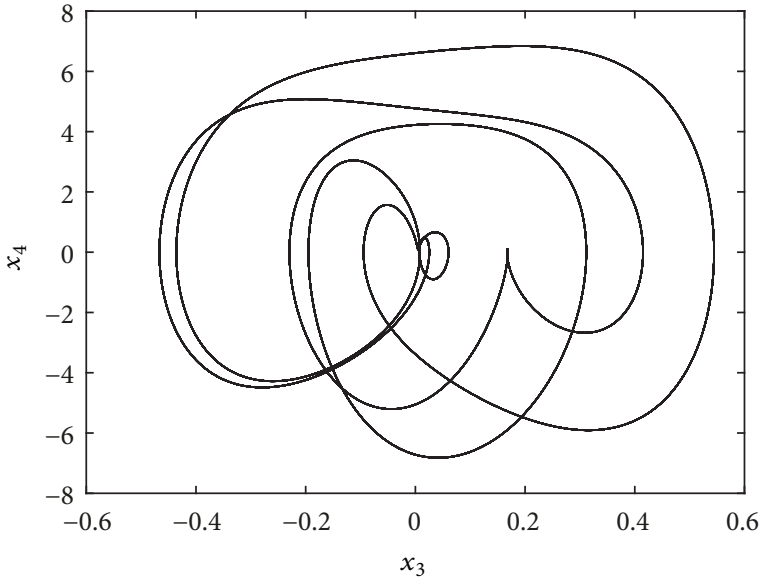

(b)

FIgURE 15: Multiperiod motions of the two modes for the trapezoidal plate under excitation amplitude $P_{0}=40 \mathrm{~N}$.

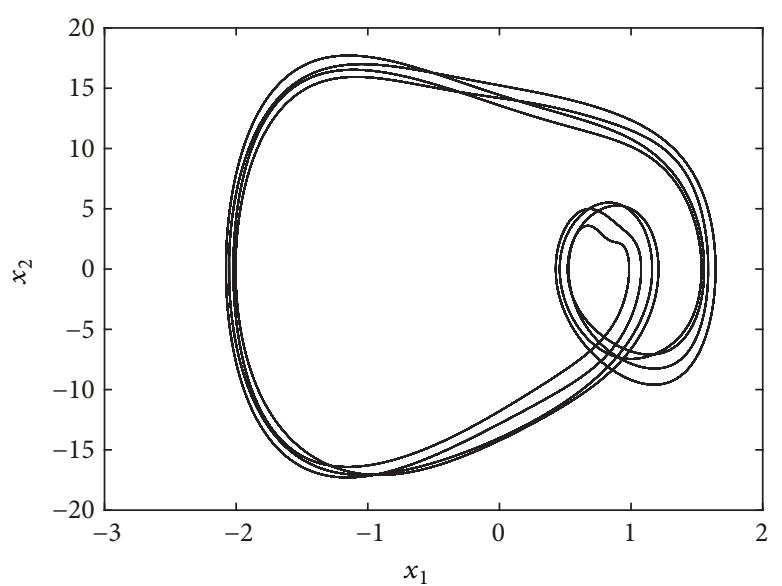

(a)

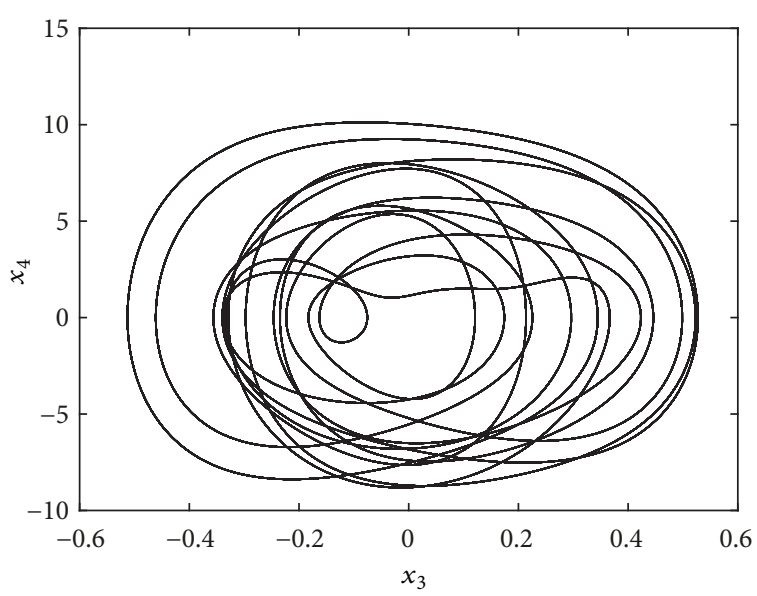

(b)

FIGURE 16: Multiperiod motions of the two modes for the trapezoidal plate under excitation amplitude $P_{0}=40.1 \mathrm{~N}$. 


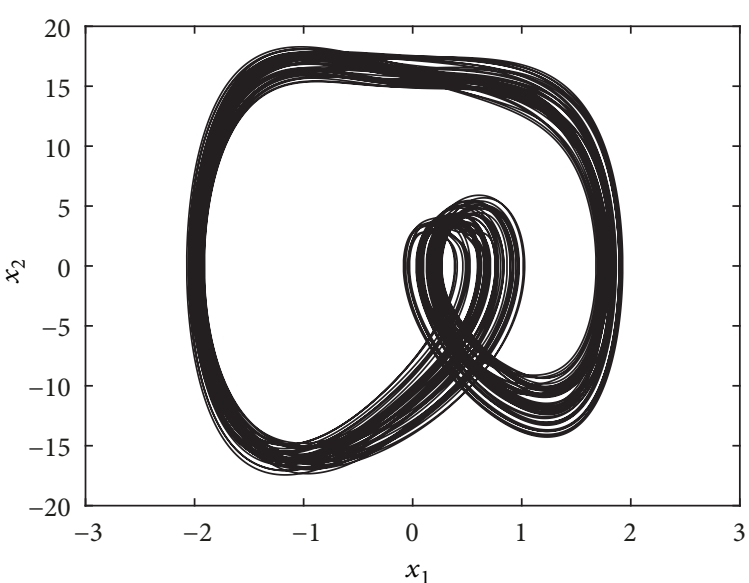

(a)

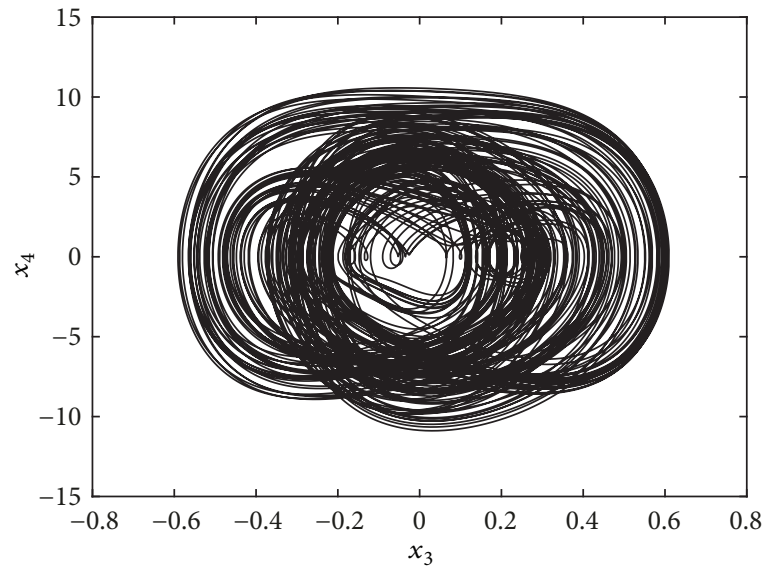

(b)

FIGURE 17: Chaotic motions of the two modes for the trapezoidal plate under excitation amplitude $P_{0}=40.5 \mathrm{~N}$.

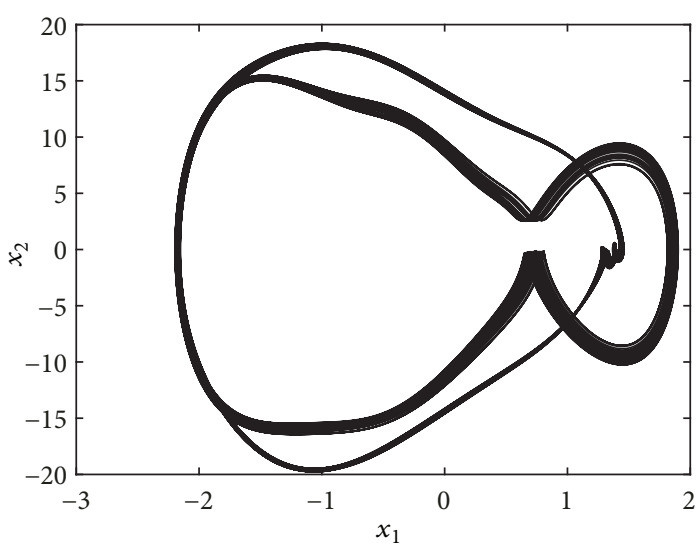

(a)

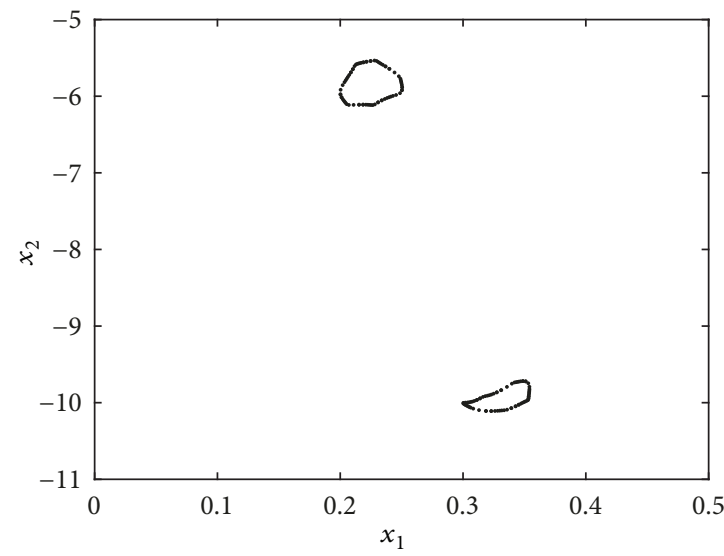

(c)

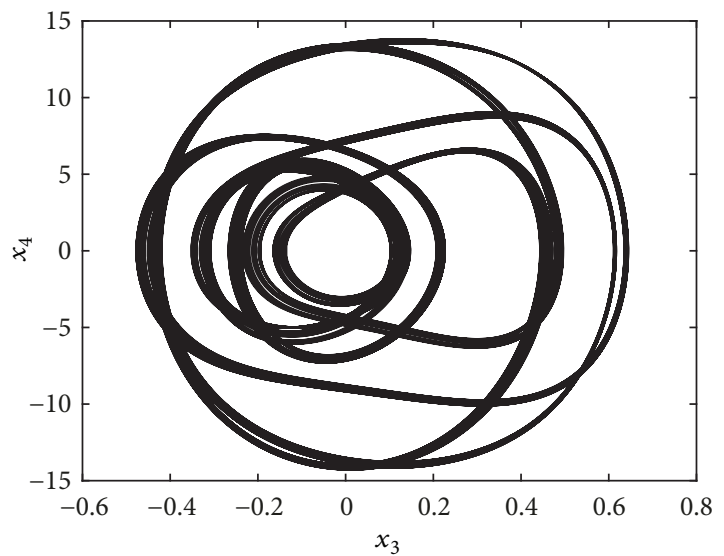

(b)

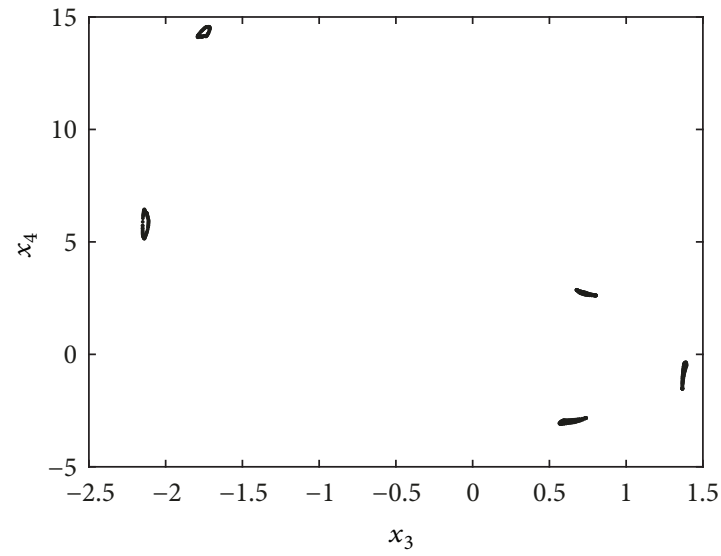

(d)

Figure 18: Quasi-periodic motions of the two modes for the trapezoidal plate under excitation amplitude $P_{0}=70 \mathrm{~N}$ : (a), (b) phase plots; (c), (d) Poincare maps. 


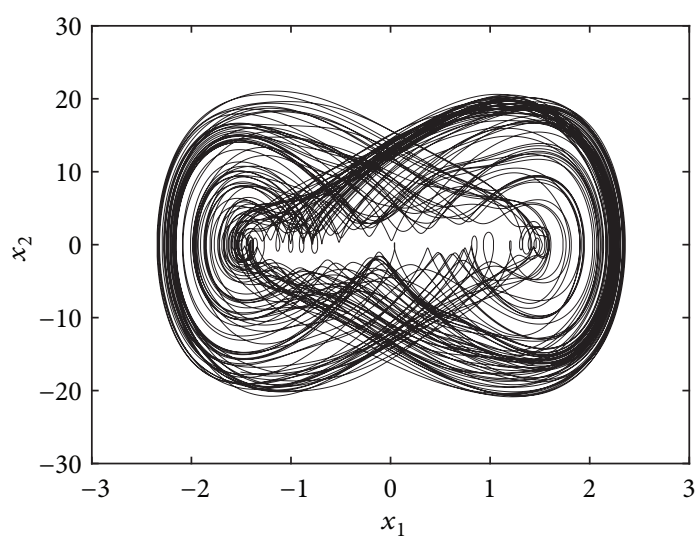

(a)

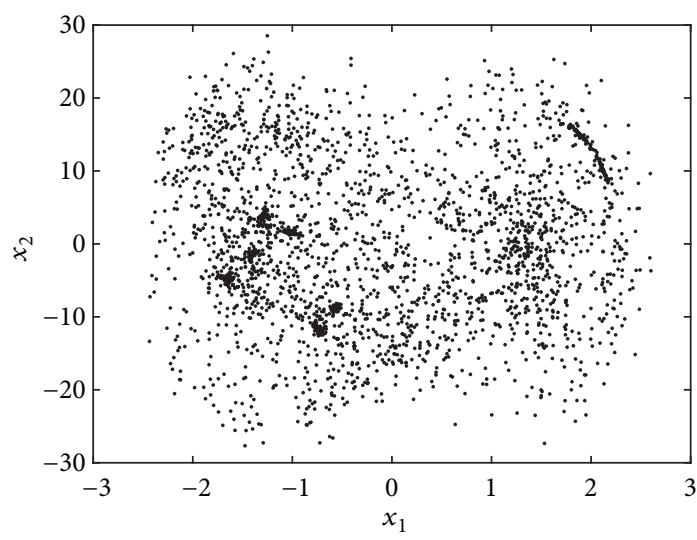

(c)

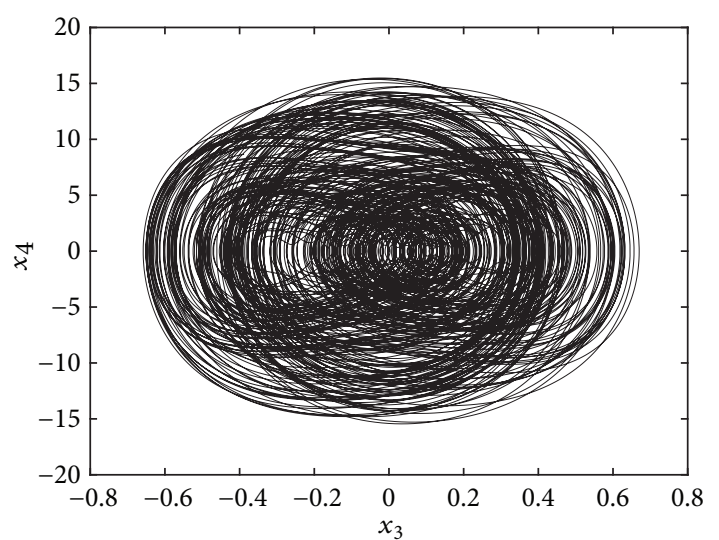

(b)

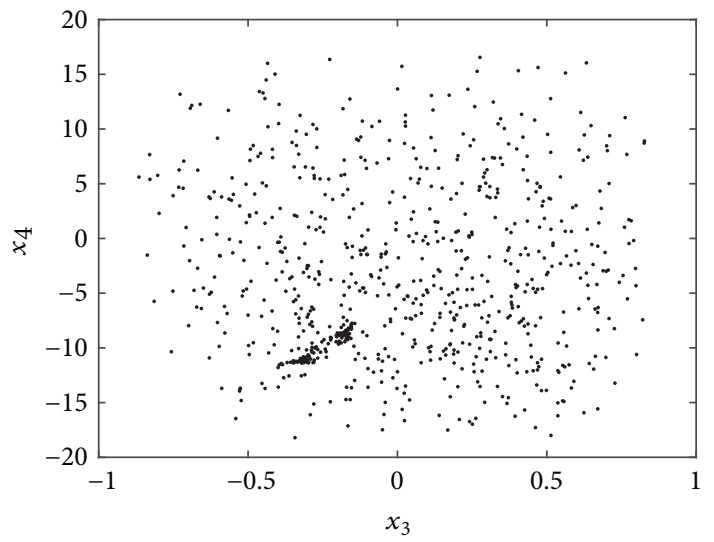

(d)

FIGURE 19: Chaotic motions of the two modes for the trapezoidal plate under excitation amplitude $P_{0}=80 \mathrm{~N}$ : (a), (b) phase plots; (c), (d) Poincare maps.

$P_{0}>4.7 \mathrm{~N}$. It demonstrates that increasing the damping level can eliminate complex responses, including multiperiod and chaotic motions.

Based on the above studies of amplitude-frequency behaviors and nonlinear dynamic responses of the trapezoidal cantilever plate with different system parameters, it is observed that there exist some adverse behaviors of the system in the case of $1: 3$ internal resonance $\omega_{2} \approx 3 \omega_{1}$, including the coupling of modes and complex dynamic responses, but these phenomena are undesirable in the structure dynamic design and engineering vibration tests. Therefore, proper excitation and system parameters should be designed to avoid undesired resonance cases.

\section{Concluding Remarks}

The Rayleigh-Ritz method and affine transformation is developed to investigate the nonlinear vibration characteristics of the trapezoidal cantilever plates with irregular geometries under transverse harmonic excitation. By changing geometrical parameters of the system, it is found that there exists the 1:3 internal resonance for the trapezoidal plate. The amplitudefrequency formulations of the truncated two-mode system in three different coupled conditions are derived by using the multiple scales method for the 1:3 internal resonance analysis. The multivalue and jump phenomena are found in the resonance region, which are clarified by the stability analysis of steady-state solutions. The effects of the system parameters, including detuning parameter and external excitation, on nonlinear vibration characteristics of the trapezoidal plate are studied. Moreover, nonlinear dynamic responses are obtained by solving the averaged equations of the system numerically. The main conclusions can be drawn as follows.

(1) The amplitude-frequency responses show that when the two modes of the system are strongly coupled, the nonlinear stiffness behavior of the second mode will be changed from hardening-spring to soft-spring characteristic.

(2) The detuning parameter and external excitation have apparent effects on the amplitude-frequency behaviors. In particular, the difference of detuning parameters can change the soft/hardening-spring characteristic. However, the excitation amplitude does not affect the nonlinear stiffness behavior and only lead to a change in resonance frequency. Meanwhile the vibration amplitude of the first mode is larger than that of the second mode, with the occurrence of internal resonance as a result of the coupling of the two modes.

(3) The stability of steady-state solutions with 1:3 internal resonance depends on the damping coefficient of the system. 
Mathematical Problems in Engineering

19

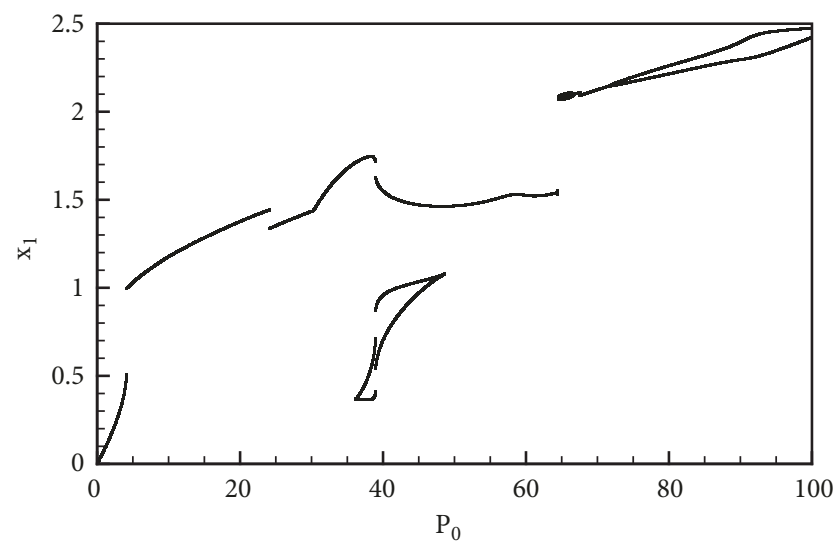

(a)

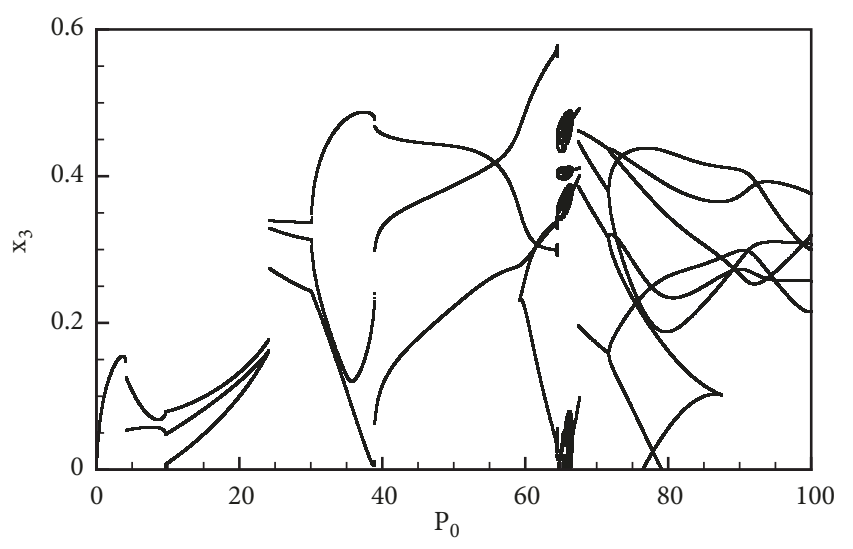

(b)

FIGURE 20: Bifurcation of the two modes for the trapezoidal plate with $\gamma_{1}=\gamma_{2}=0.1$.

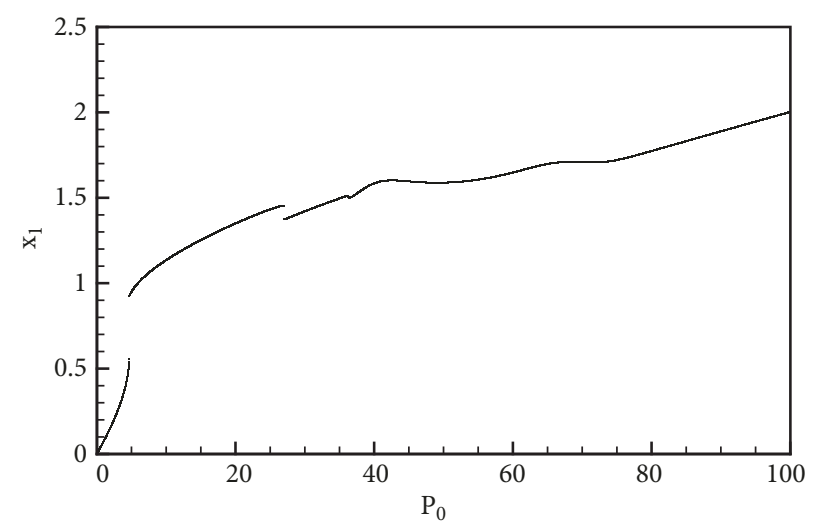

(a)

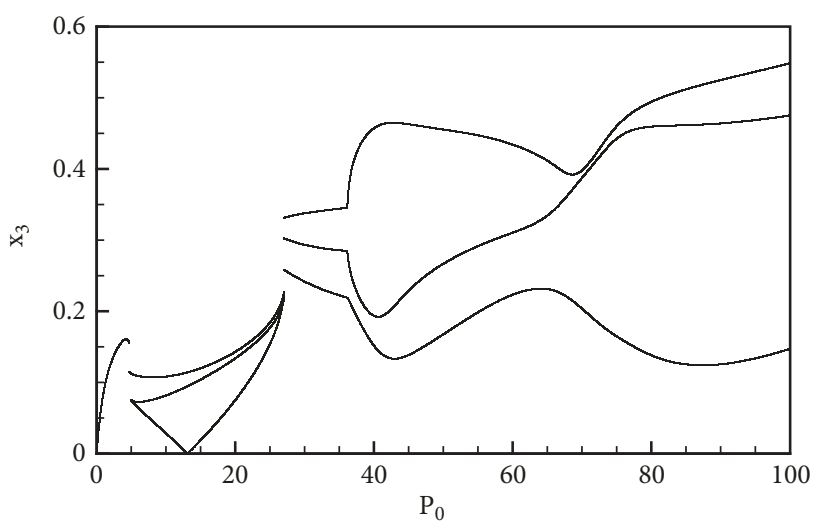

(b)

FIGURE 21: Bifurcation of the two modes for the trapezoidal plate with $\gamma_{1}=\gamma_{2}=0.2$.

The numerical results show that the excitation amplitude has significant influence on nonlinear dynamic response. There exist the periodic, quasi-periodic, and chaotic motions and the route to chaos is via period-doubling for the present trapezoidal plate with the 1:3 internal resonance case.

(4) The obtained results demonstrate that appropriate excitation and system parameters can be designed to avoid undesired resonance and possible adverse dynamic behaveions.

Appendix

$$
\begin{gathered}
F_{q_{1}^{3}, i}=\int_{0}^{1} \phi_{1}^{\prime} \phi_{1}^{\prime} \phi_{1}^{\prime} \phi_{i}^{\prime} d \xi \int_{0}^{1} \psi_{1} \psi_{1} \psi_{1} \psi_{1} H^{4} J^{\prime} d \eta+\left(\frac{c_{r}}{l}\right)^{2} \\
\cdot\left[2 \int_{0}^{1} \phi_{1}^{\prime} \phi_{1}^{\prime} \phi_{1}^{\prime} \phi_{i}^{\prime} G^{2} d \xi \int_{0}^{1} \psi_{1} \psi_{1} \psi_{1} \psi_{1} H^{4} J^{\prime} d \eta\right. \\
+\int_{0}^{1} \phi_{1}^{\prime} \phi_{1}^{\prime} \phi_{1}^{\prime} \phi_{i} G d \xi \int_{0}^{1} \psi_{1} \psi_{1} \psi_{1} \psi_{1}^{\prime} H^{3} J^{\prime} d \eta
\end{gathered}
$$

$$
\begin{aligned}
& +3 \int_{0}^{1} \phi_{1}^{\prime} \phi_{1}^{\prime} \phi_{1} \phi_{i}^{\prime} G d \xi \int_{0}^{1} \psi_{1} \psi_{1} \psi_{1} \psi_{1}^{\prime} H^{3} J^{\prime} d \eta \\
& +\int_{0}^{1} \phi_{1}^{\prime} \phi_{1}^{\prime} \phi_{1} \phi_{i} d \xi \int_{0}^{1} \psi_{1} \psi_{1} \psi_{1}^{\prime} \psi_{1}^{\prime} H^{2} J^{\prime} d \eta \\
& \left.+\int_{0}^{1} \phi_{1} \phi_{1} \phi_{1}^{\prime} \phi_{i}^{\prime} d \xi \int_{0}^{1} \psi_{1} \psi_{1} \psi_{1}^{\prime} \psi_{1}^{\prime} H^{2} J^{\prime} d \eta\right]+\left(\frac{c_{r}}{l}\right)^{4} \\
& +\left[\int_{0}^{1} \phi_{1}^{\prime} \phi_{1}^{\prime} \phi_{1}^{\prime} \phi_{i}^{\prime} G^{4} d \xi \int_{0}^{1} \psi_{1} \psi_{1} \psi_{1} \psi_{1} H^{4} J^{\prime} d \eta\right. \\
& +\int_{0}^{1} \phi_{1}^{\prime} \phi_{1}^{\prime} \phi_{1}^{\prime} \phi_{i} G^{3} d \xi \int_{0}^{1} \psi_{1} \psi_{1} \psi_{1} \psi_{1}^{\prime} H^{3} J^{\prime} d \eta \\
& +3 \int_{0}^{1} \phi_{1}^{\prime} \phi_{1}^{\prime} \phi_{1} \phi_{i}^{\prime} G^{3} d \xi \int_{0}^{1} \psi_{1} \psi_{1} \psi_{1} \psi_{1}^{\prime} H^{3} J^{\prime} d \eta \\
& +3 \int_{0}^{1} \phi_{1}^{\prime} \phi_{1}^{\prime} \phi_{1} \phi_{i} G^{2} d \xi \int_{0}^{1} \psi_{1} \psi_{1} \psi_{1}^{\prime} \psi_{1}^{\prime} H^{2} J^{\prime} d \eta \\
& +3 \int_{0}^{1} \phi_{1}^{\prime} \phi_{1} \phi_{1} \phi_{i}^{\prime} G^{2} d \xi \int_{0}^{1} \psi_{1} \psi_{1} \psi_{1}^{\prime} \psi_{1}^{\prime} H^{2} J^{\prime} d \eta
\end{aligned}
$$


20

Mathematical Problems in Engineering

$$
\begin{aligned}
& +3 \int_{0}^{1} \phi_{1}^{\prime} \phi_{1} \phi_{1} \phi_{i} G d \xi \int_{0}^{1} \psi_{1} \psi_{1}^{\prime} \psi_{1}^{\prime} \psi_{1}^{\prime} H J^{\prime} d \eta \\
& +\int_{0}^{1} \phi_{1} \phi_{1} \phi_{1} \phi_{i}^{\prime} d \xi \int_{0}^{1} \psi_{1} \psi_{1}^{\prime} \psi_{1}^{\prime} \psi_{1}^{\prime} J^{\prime} d \eta \\
& \left.+\int_{0}^{1} \phi_{1} \phi_{1} \phi_{1} \phi_{i} d \xi \int_{0}^{1} \psi_{1}^{\prime} \psi_{1}^{\prime} \psi_{1}^{\prime} \psi_{1}^{\prime} J^{\prime} d \eta\right] \\
& F_{q_{1}^{2} q_{2}, i}=3 \int_{0}^{1} \phi_{1}^{\prime} \phi_{1}^{\prime} \phi_{2}^{\prime} \phi_{i}^{\prime} d \xi \int_{0}^{1} \psi_{1} \psi_{1} \psi_{1} \psi_{1} H^{4} J^{\prime} d \eta \\
& +\left(\frac{c_{r}}{l}\right)^{2} \\
& \cdot\left[6 \int_{0}^{1} \phi_{1}^{\prime} \phi_{1}^{\prime} \phi_{2}^{\prime} \phi_{i}^{\prime} G^{2} d \xi \int_{0}^{1} \psi_{1} \psi_{1} \psi_{1} \psi_{1} H^{4} J^{\prime} d \eta\right. \\
& +3 \int_{0}^{1} \phi_{1}^{\prime} \phi_{1}^{\prime} \phi_{2}^{\prime} \phi_{i} G d \xi \int_{0}^{1} \psi_{1} \psi_{1} \psi_{1} \psi_{1}^{\prime} H^{3} J^{\prime} d \eta \\
& +6 \int_{0}^{1} \phi_{1}^{\prime} \phi_{1} \phi_{2}^{\prime} \phi_{i}^{\prime} G d \xi \int_{0}^{1} \psi_{1} \psi_{1} \psi_{1} \psi_{1}^{\prime} H^{3} J^{\prime} d \eta \\
& +3 \int_{0}^{1} \phi_{1}^{\prime} \phi_{1}^{\prime} \phi_{2} \phi_{i}^{\prime} G d \xi \int_{0}^{1} \psi_{1} \psi_{1} \psi_{1} \psi_{1}^{\prime} H^{3} J^{\prime} d \eta \\
& +2 \int_{0}^{1} \phi_{1}^{\prime} \phi_{1} \phi_{2}^{\prime} \phi_{i} d \xi \int_{0}^{1} \psi_{1} \psi_{1} \psi_{1}^{\prime} \psi_{1}^{\prime} H^{2} J^{\prime} d \eta \\
& +\int_{0}^{1} \phi_{1}^{\prime} \phi_{1}^{\prime} \phi_{2} \phi_{i} d \xi \int_{0}^{1} \psi_{1} \psi_{1} \psi_{1}^{\prime} \psi_{1}^{\prime} H^{2} J^{\prime} d \eta \\
& \cdot+2 \int_{0}^{1} \phi_{1}^{\prime} \phi_{1} \phi_{2} \phi_{i}^{\prime} d \xi \int_{0}^{1} \psi_{1} \psi_{1} \psi_{1}^{\prime} \psi_{1}^{\prime} H^{2} J^{\prime} d \eta \\
& \left.+\int_{0}^{1} \phi_{1} \phi_{1} \phi_{2}^{\prime} \phi_{i}^{\prime} d \xi \int_{0}^{1} \psi_{1} \psi_{1} \psi_{1}^{\prime} \psi_{1}^{\prime} H^{2} J^{\prime} d \eta\right]+\left(\frac{c_{r}}{l}\right)^{4} \\
& \cdot\left[3 \int_{0}^{1} \phi_{1}^{\prime} \phi_{1}^{\prime} \phi_{2}^{\prime} \phi_{i}^{\prime} G^{4} d \xi \int_{0}^{1} \psi_{1} \psi_{1} \psi_{1} \psi_{1} H^{4} J^{\prime} d \eta\right. \\
& +3 \int_{0}^{1} \phi_{1}^{\prime} \phi_{1}^{\prime} \phi_{2}^{\prime} \phi_{i} G^{3} d \xi \int_{0}^{1} \psi_{1} \psi_{1} \psi_{1} \psi_{1}^{\prime} H^{3} J^{\prime} d \eta \\
& +6 \int_{0}^{1} \phi_{1}^{\prime} \phi_{1} \phi_{2}^{\prime} \phi_{i}^{\prime} G^{3} d \xi \int_{0}^{1} \psi_{1} \psi_{1} \psi_{1} \psi_{1}^{\prime} H^{3} J^{\prime} d \eta \\
& +3 \int_{0}^{1} \phi_{1}^{\prime} \phi_{1}^{\prime} \phi_{2} \phi_{i}^{\prime} G^{3} d \xi \int_{0}^{1} \psi_{1} \psi_{1} \psi_{1} \psi_{1}^{\prime} H^{3} J^{\prime} d \eta \\
& +6 \int_{0}^{1} \phi_{1}^{\prime} \phi_{1} \phi_{2}^{\prime} \phi_{i} G^{2} d \xi \int_{0}^{1} \psi_{1} \psi_{1} \psi_{1}^{\prime} \psi_{1}^{\prime} H^{2} J^{\prime} d \eta \\
& +3 \int_{0}^{1} \phi_{1}^{\prime} \phi_{1}^{\prime} \phi_{2} \phi_{i} G^{2} d \xi \int_{0}^{1} \psi_{1} \psi_{1} \psi_{1}^{\prime} \psi_{1}^{\prime} H^{2} J^{\prime} d \eta \\
& +6 \int_{0}^{1} \phi_{1}^{\prime} \phi_{1} \phi_{2} \phi_{i}^{\prime} G^{2} d \xi \int_{0}^{1} \psi_{1} \psi_{1} \psi_{1}^{\prime} \psi_{1}^{\prime} H^{2} J^{\prime} d \eta \\
& +3 \int_{0}^{1} \phi_{1}^{\prime} \phi_{1}^{\prime} \phi_{2} \phi_{i} G^{2} d \xi \int_{0}^{1} \psi_{1} \psi_{1} \psi_{1}^{\prime} \psi_{1}^{\prime} H^{2} J^{\prime} d \eta
\end{aligned}
$$

$$
\begin{aligned}
& +6 \int_{0}^{1} \phi_{1}^{\prime} \phi_{1} \phi_{2} \phi_{i} G d \xi \int_{0}^{1} \psi_{1} \psi_{1}^{\prime} \psi_{1}^{\prime} \psi_{1}^{\prime} H J^{\prime} d \eta \\
& +3 \int_{0}^{1} \phi_{1} \phi_{1} \phi_{2}^{\prime} \phi_{i} G d \xi \int_{0}^{1} \psi_{1} \psi_{1}^{\prime} \psi_{1}^{\prime} \psi_{1}^{\prime} H J^{\prime} d \eta \\
& +3 \int_{0}^{1} \phi_{1} \phi_{1} \phi_{2} \phi_{i}^{\prime} G d \xi \int_{0}^{1} \psi_{1} \psi_{1}^{\prime} \psi_{1}^{\prime} \psi_{1}^{\prime} H J^{\prime} d \eta \\
& \left.+\int_{0}^{1} \phi_{1} \phi_{1} \phi_{2} \phi_{i} d \xi \int_{0}^{1} \psi_{1}^{\prime} \psi_{1}^{\prime} \psi_{1}^{\prime} \psi_{1}^{\prime} J^{\prime} d \eta\right]
\end{aligned}
$$

(A.1)

$$
F_{q_{1} q_{2}^{2}, i}=3 \int_{0}^{1} \phi_{1}^{\prime} \phi_{2}^{\prime} \phi_{2}^{\prime} \phi_{i}^{\prime} d \xi \int_{0}^{1} \psi_{1} \psi_{1} \psi_{1} \psi_{1} H^{4} J^{\prime} d \eta
$$$$
+\left(\frac{c_{r}}{l}\right)^{2}
$$

$$
\text { . }\left[6 \int_{0}^{1} \phi_{1}^{\prime} \phi_{2}^{\prime} \phi_{2}^{\prime} \phi_{i}^{\prime} G^{2} d \xi \int_{0}^{1} \psi_{1} \psi_{1} \psi_{1} \psi_{1} H^{4} J^{\prime} d \eta\right.
$$$$
+3 \int_{0}^{1} \phi_{1}^{\prime} \phi_{2}^{\prime} \phi_{2}^{\prime} \phi_{i} G d \xi \int_{0}^{1} \psi_{1} \psi_{1} \psi_{1} \psi_{1}^{\prime} H^{3} J^{\prime} d \eta
$$$$
+6 \int_{0}^{1} \phi_{1}^{\prime} \phi_{2}^{\prime} \phi_{2} \phi_{i}^{\prime} G d \xi \int_{0}^{1} \psi_{1} \psi_{1} \psi_{1} \psi_{1}^{\prime} H^{3} J^{\prime} d \eta
$$$$
+3 \int_{0}^{1} \phi_{1} \phi_{2}^{\prime} \phi_{2}^{\prime} \phi_{i}^{\prime} G d \xi \int_{0}^{1} \psi_{1} \psi_{1} \psi_{1} \psi_{1}^{\prime} H^{3} J^{\prime} d \eta
$$$$
+2 \int_{0}^{1} \phi_{1}^{\prime} \phi_{2}^{\prime} \phi_{2} \phi_{i} d \xi \int_{0}^{1} \psi_{1} \psi_{1} \psi_{1}^{\prime} \psi_{1}^{\prime} H^{2} J^{\prime} d \eta
$$$$
+\int_{0}^{1} \phi_{1} \phi_{2}^{\prime} \phi_{2}^{\prime} \phi_{i} d \xi \int_{0}^{1} \psi_{1} \psi_{1} \psi_{1}^{\prime} \psi_{1}^{\prime} H^{2} J^{\prime} d \eta
$$$$
+2 \int_{0}^{1} \phi_{1} \phi_{2} \phi_{2}^{\prime} \phi_{i}^{\prime} d \xi \int_{0}^{1} \psi_{1} \psi_{1} \psi_{1}^{\prime} \psi_{1}^{\prime} H^{2} J^{\prime} d \eta
$$$$
\left.+\int_{0}^{1} \phi_{1}^{\prime} \phi_{2} \phi_{2} \phi_{i}^{\prime} d \xi \int_{0}^{1} \psi_{1} \psi_{1} \psi_{1}^{\prime} \psi_{1}^{\prime} H^{2} J^{\prime} d \eta\right]+\left(\frac{c_{r}}{l}\right)^{4}
$$

$\cdot\left[3 \int_{0}^{1} \phi_{1}^{\prime} \phi_{2}^{\prime} \phi_{2}^{\prime} \phi_{i}^{\prime} G^{4} d \xi \int_{0}^{1} \psi_{1} \psi_{1} \psi_{1} \psi_{1} H^{4} J^{\prime} d \eta\right.$

$+3 \int_{0}^{1} \phi_{1}^{\prime} \phi_{2}^{\prime} \phi_{2}^{\prime} \phi_{i} G^{3} d \xi \int_{0}^{1} \psi_{1} \psi_{1} \psi_{1} \psi_{1}^{\prime} H^{3} J^{\prime} d \eta$

$+6 \int_{0}^{1} \phi_{1}^{\prime} \phi_{2}^{\prime} \phi_{2} \phi_{i}^{\prime} G^{3} d \xi \int_{0}^{1} \psi_{1} \psi_{1} \psi_{1} \psi_{1}^{\prime} H^{3} J^{\prime} d \eta$

$+3 \int_{0}^{1} \phi_{1} \phi_{2}^{\prime} \phi_{2}^{\prime} \phi_{i}^{\prime} G^{3} d \xi \int_{0}^{1} \psi_{1} \psi_{1} \psi_{1} \psi_{1}^{\prime} H^{3} J^{\prime} d \eta$

$+6 \int_{0}^{1} \phi_{1}^{\prime} \phi_{2}^{\prime} \phi_{1} \phi_{i} G^{2} d \xi \int_{0}^{1} \psi_{1} \psi_{1} \psi_{1}^{\prime} \psi_{1}^{\prime} H^{2} J^{\prime} d \eta$

$+3 \int_{0}^{1} \phi_{1} \phi_{2}^{\prime} \phi_{2}^{\prime} \phi_{i} G^{2} d \xi \int_{0}^{1} \psi_{1} \psi_{1} \psi_{1}^{\prime} \psi_{1}^{\prime} H^{2} J^{\prime} d \eta$

$+6 \int_{0}^{1} \phi_{1} \phi_{2} \phi_{2}^{\prime} \phi_{i}^{\prime} G^{2} d \xi \int_{0}^{1} \psi_{1} \psi_{1} \psi_{1}^{\prime} \psi_{1}^{\prime} H^{2} J^{\prime} d \eta$ 


$$
\begin{aligned}
& +3 \int_{0}^{1} \phi_{1} \phi_{2}^{\prime} \phi_{2}^{\prime} \phi_{i} G^{2} d \xi \int_{0}^{1} \psi_{1} \psi_{1} \psi_{1}^{\prime} \psi_{1}^{\prime} H^{2} J^{\prime} d \eta \\
& +6 \int_{0}^{1} \phi_{1} \phi_{2} \phi_{2}^{\prime} \phi_{i} G d \xi \int_{0}^{1} \psi_{1} \psi_{1}^{\prime} \psi_{1}^{\prime} \psi_{1}^{\prime} H J^{\prime} d \eta \\
& +3 \int_{0}^{1} \phi_{1}^{\prime} \phi_{2} \phi_{2} \phi_{i} G d \xi \int_{0}^{1} \psi_{1} \psi_{1}^{\prime} \psi_{1}^{\prime} \psi_{1}^{\prime} H J^{\prime} d \eta \\
& +3 \int_{0}^{1} \phi_{1} \phi_{2} \phi_{2} \phi_{i}^{\prime} G d \xi \int_{0}^{1} \psi_{1} \psi_{1}^{\prime} \psi_{1}^{\prime} \psi_{1}^{\prime} H J^{\prime} d \eta \\
& \left.+\int_{0}^{1} \phi_{1} \phi_{2} \phi_{2} \phi_{i} d \xi \int_{0}^{1} \psi_{1}^{\prime} \psi_{1}^{\prime} \psi_{1}^{\prime} \psi_{1}^{\prime} J^{\prime} d \eta\right] \\
& F_{q_{2}^{3}, i}=\int_{0}^{1} \phi_{2}^{\prime} \phi_{2}^{\prime} \phi_{2}^{\prime} \phi_{i}^{\prime} d \xi \int_{0}^{1} \psi_{1} \psi_{1} \psi_{1} \psi_{1} H^{4} J^{\prime} d \eta+\left(\frac{c_{r}}{l}\right)^{2} \\
& \cdot\left[2 \int_{0}^{1} \phi_{2}^{\prime} \phi_{2}^{\prime} \phi_{2}^{\prime} \phi_{i}^{\prime} G^{2} d \xi \int_{0}^{1} \psi_{1} \psi_{1} \psi_{1} \psi_{1} H^{4} J^{\prime} d \eta\right. \\
& +\int_{0}^{1} \phi_{2}^{\prime} \phi_{2}^{\prime} \phi_{2}^{\prime} \phi_{i} G d \xi \int_{0}^{1} \psi_{1} \psi_{1} \psi_{1} \psi_{1}^{\prime} H^{3} J^{\prime} d \eta \\
& +3 \int_{0}^{1} \phi_{2}^{\prime} \phi_{2}^{\prime} \phi_{2} \phi_{i}^{\prime} G d \xi \int_{0}^{1} \psi_{1} \psi_{1} \psi_{1} \psi_{1}^{\prime} H^{3} J^{\prime} d \eta \\
& +\int_{0}^{1} \phi_{2}^{\prime} \phi_{2}^{\prime} \phi_{2} \phi_{i} d \xi \int_{0}^{1} \psi_{1} \psi_{1} \psi_{1}^{\prime} \psi_{1}^{\prime} H^{2} J^{\prime} d \eta \\
& \left.+\int_{0}^{1} \phi_{2} \phi_{2} \phi_{2}^{\prime} \phi_{i}^{\prime} d \xi \int_{0}^{1} \psi_{1} \psi_{1} \psi_{1}^{\prime} \psi_{1}^{\prime} H^{2} J^{\prime} d \eta\right]+\left(\frac{c_{r}}{l}\right)^{4} \\
& \cdot\left[\int_{0}^{1} \phi_{2}^{\prime} \phi_{2}^{\prime} \phi_{2}^{\prime} \phi_{i}^{\prime} G^{4} d \xi \int_{0}^{1} \psi_{1} \psi_{1} \psi_{1} \psi_{1} H^{4} J^{\prime} d \eta\right. \\
& +\int_{0}^{1} \phi_{2}^{\prime} \phi_{2}^{\prime} \phi_{2}^{\prime} \phi_{i} G^{3} d \xi \int_{0}^{1} \psi_{1} \psi_{1} \psi_{1} \psi_{1}^{\prime} H^{3} J^{\prime} d \eta \\
& +3 \int_{0}^{1} \phi_{2}^{\prime} \phi_{2}^{\prime} \phi_{2} \phi_{i}^{\prime} G^{3} d \xi \int_{0}^{1} \psi_{1} \psi_{1} \psi_{1} \psi_{1}^{\prime} H^{3} J^{\prime} d \eta \\
& +3 \int_{0}^{1} \phi_{2}^{\prime} \phi_{2}^{\prime} \phi_{2} \phi_{i} G^{2} d \xi \int_{0}^{1} \psi_{1} \psi_{1} \psi_{1}^{\prime} \psi_{1}^{\prime} H^{2} J^{\prime} d \eta \\
& +3 \int_{0}^{1} \phi_{2}^{\prime} \phi_{2} \phi_{2} \phi_{i}^{\prime} G^{2} d \xi \int_{0}^{1} \psi_{1} \psi_{1} \psi_{1}^{\prime} \psi_{1}^{\prime} H^{2} J^{\prime} d \eta \\
& +3 \int_{0}^{1} \phi_{2}^{\prime} \phi_{2} \phi_{2} \phi_{i} G d \xi \int_{0}^{1} \psi_{1} \psi_{1}^{\prime} \psi_{1}^{\prime} \psi_{1}^{\prime} H J^{\prime} d \eta \\
& +\int_{0}^{1} \phi_{2} \phi_{2} \phi_{2} \phi_{i}^{\prime} d \xi \int_{0}^{1} \psi_{1} \psi_{1}^{\prime} \psi_{1}^{\prime} \psi_{1}^{\prime} J^{\prime} d \eta \\
& \left.+\int_{0}^{1} \phi_{2} \phi_{2} \phi_{2} \phi_{i} d \xi \int_{0}^{1} \psi_{1}^{\prime} \psi_{1}^{\prime} \psi_{1}^{\prime} \psi_{1}^{\prime} J^{\prime} d \eta\right]
\end{aligned}
$$

\begin{tabular}{|c|c|}
\hline$c_{r}, c_{t}:$ & Root length and tip length, respectively \\
\hline$D:$ & Plate stiffness, $D=E h^{3} / 12\left(1-v^{2}\right)$ \\
\hline$E:$ & Young's modulus \\
\hline$h:$ & Plate thickness \\
\hline$l:$ & The distance between root side and tip side \\
\hline$I, J:$ & $\begin{array}{l}\text { Total mode number retained in the } \xi \text { and } \\
\eta \text { directions for in-plane displacement } u \text {, } \\
\text { respectively }\end{array}$ \\
\hline$i, j:$ & $\begin{array}{l}\text { Mode number retained in the } \xi \text { and } \eta \\
\text { directions for in-plane displacement } u \text {, } \\
\text { respectively }\end{array}$ \\
\hline$L=T-U:$ & Lagrangian \\
\hline$M, N:$ & $\begin{array}{l}\text { Total mode number retained in the } \xi \text { and } \\
\eta \text { directions for transverse deflection } w \text {, } \\
\text { respectively }\end{array}$ \\
\hline$m, n:$ & $\begin{array}{l}\text { Mode number retained in the } \xi \text { and } \eta \\
\text { directions for transverse deflection } w \text {, } \\
\text { respectively }\end{array}$ \\
\hline$q_{m n}:$ & $\begin{array}{l}\text { Mode coordinate for transverse deflection } \\
w\end{array}$ \\
\hline$R, S:$ & $\begin{array}{l}\text { Total mode number retained in the } \xi \text { and } \\
\eta \text { directions for in-plane displacement } v \text {, } \\
\text { respectively }\end{array}$ \\
\hline$r, s:$ & $\begin{array}{l}\text { Mode number retained in the } \xi \text { and } \eta \\
\text { directions for in-plane displacement } v \text {, } \\
\text { respectively }\end{array}$ \\
\hline$P_{0}:$ & Excitation amplitude \\
\hline$T:$ & Kinetic energy \\
\hline$t, T_{0}, T_{1}:$ & Time \\
\hline$U:$ & Elastic energy \\
\hline$u, v:$ & $\begin{array}{l}\text { In-plane displacement in the } \xi \text { and } \eta \\
\text { directions, respectively }\end{array}$ \\
\hline $\bar{u}, \bar{v}:$ & $\begin{array}{l}\text { Nondimensional in-plane displacement in } \\
\text { the } \xi \text { and } \eta \text { directions, respectively }\end{array}$ \\
\hline$u_{i(r)}, v_{j(s)}$ & $\begin{array}{l}\text { Mode in the } \xi \text { and } \eta \text { directions for } \\
\text { in-plane displacement } u(v) \text {, respectively }\end{array}$ \\
\hline$w:$ & Transverse deflection \\
\hline $\bar{w}:$ & Nondimensional transverse deflection \\
\hline$x, y, z:$ & Physical coordinates \\
\hline$\gamma_{1}, \gamma_{2}$ & Modal damping ratios \\
\hline$v:$ & Poisson ratio \\
\hline$\rho_{m}:$ & Plate density \\
\hline$\xi, \eta:$ & Nondimensional coordinates \\
\hline$\xi_{e}, \eta_{e}$ & Location coordinates of excitation point \\
\hline$\tau:$ & Nondimensional time, $\tau=t\left(D / \rho_{m} h c_{r}^{4}\right)^{1 / 2}$ \\
\hline$\omega_{1}, \omega_{2}:$ & Natural frequencies of the first two modes \\
\hline$\sigma_{1}, \sigma_{2}:$ & Detuning parameters \\
\hline$\Omega_{0}:$ & Excitation frequency \\
\hline()$^{\prime}:$ & $d() / d \xi$ or $d() / d \eta$ \\
\hline()$^{\prime \prime}:$ & $d^{2}() / d \xi^{2}$ or $d^{2}() / d \eta^{2}$ \\
\hline$(\cdot):$ & $d() / d \tau$ \\
\hline
\end{tabular}

\section{Nomenclature}

\footnotetext{
$a_{i j}, b_{r s}$ : Mode coordinate for in-plane displacement $u$ and $v$, respectively
}

\section{Data Availability}

The data used to support the findings of this study are available from the corresponding author upon request. 


\section{Conflicts of Interest}

The authors declare that there are no conflicts of interest regarding the publication of this paper.

\section{Acknowledgments}

This work is supported by the Special Financial Grant from the China Postdoctoral Science Foundation (Grant No. 2019TQ0253) and National Key Laboratory of Science and Technology on Liquid Rocket Engine of Xian Aerospace Propulsion Institute.

\section{References}

[1] E. H. Dowell, "Panel flutter-a review of the aeroelastic stability of plates and shells," AIAA Journal, vol. 8, no. 3, pp. 385-399, 1970.

[2] D. Xie, M. Xu, H. Dai, and E. H. Dowell, "Proper orthogonal decomposition method for analysis of nonlinear panel flutter with thermal effects in supersonic flow," Journal of Sound and Vibration, vol. 337, pp. 263-283, 2015.

[3] X. Wang, Z. Yang, W. Wang, and W. Tian, "Nonlinear viscoelastic heated panel flutter with aerodynamic loading exerted on both surfaces," Journal of Sound and Vibration, vol. 409, pp. 306-317, 2017.

[4] O. Doaré and S. Michelin, "Piezoelectric coupling in energyharvesting fluttering flexible plates: Linear stability analysis and conversion efficiency," Journal of Fluids and Structures, vol. 27, no. 8, pp. 1357-1375, 2011.

[5] X. Wang, Z. Yang, J. Zhou, and W. Hu, "Aeroelastic effect on aerothermoacoustic response of metallic panels in supersonic flow, Chinese Journal of Aeronautics, vol. 29, no. 6, pp. 16351648, 2016.

[6] A. Shooshtari and S. Razavi, "Linear and nonlinear free vibration of a multilayered magneto-electro-elastic doubly-curved shell on elastic foundation," Composites Part B: Engineering, vol. 78, pp. 95-108, 2015.

[7] W. Tian, Z. Yang, and T. Zhao, "Nonlinear aeroelastic characteristics of an all-movable fin with freeplay and aerodynamic nonlinearities in hypersonic flow," International Journal of NonLinear Mechanics, vol. 116, pp. 123-139, 2019.

[8] Y. X. Hao, L. H. Chen, W. Zhang, and J. G. Lei, "Nonlinear oscillations, bifurcations and chaos of functionally graded materials plate," Journal of Sound and Vibration, vol. 312, no. 4-5, pp. 862-892, 2008.

[9] Y. Hao, W. Zhang, and J. Yang, "Nonlinear oscillation of a cantilever FGM rectangular plate based on third-order plate theory and asymptotic perturbation method," Composites Part B: Engineering, vol. 42, no. 3, pp. 402-413, 2011.

[10] W. Zhang, "Global and chaotic dynamics for a parametrically excited thin plate," Journal of Sound and Vibration, vol. 239, no. 5, pp. 1013-1036, 2001.

[11] W. Zhang, Z. Liu, and P. Yu, "Global dynamics of a parametrically and externally excited thin plate," Nonlinear Dynamics, vol. 24, no. 3, pp. 245-268, 2001.

[12] F. X. An and F. Q. Chen, "Multipulse orbits and chaotic dynamics of an aero-elastic fgp plate under parametric and primary excitations," International Journal of Bifurcation and Chaos, vol. 27, no. 4, article 1750050, 2017.
[13] G. Anlas and O. Elbeyli, "Nonlinear vibrations of a simply supported rectangular metallic plate subjected to transverse harmonic excitation in the presence of a one-to-one internal resonance," Nonlinear Dynamics, vol. 30, no. 1, pp. 1-28, 2002.

[14] M. Sayed, Y. S. Hamed, and Y. A. Amer, "Vibration reduction and stability of non-linear system subjected to external and parametric excitation forces under a non-linear absorber," International Journal of Contemporary Mathematical Sciences, vol. 6, no. 21-24, pp. 1051-1070, 2011.

[15] W. L. Ye and E. H. Dowell, "Limit cycle oscillation of a fluttering cantilever plate," AIAA Journal, vol. 29, no. 11, pp. 1929-1936, 2011.

[16] D. Xie, M. Xu, H. H. Dai, and E. H. Dowell, "Observation and evolution of chaos for a cantilever plate in supersonic flow," Journal of Fluids and Structures, vol. 50, pp. 271-291, 2014.

[17] D. Xie, M. Xu, H. Dai, and T. Chen, "New look at nonlinear aerodynamics in analysis of hypersonic panel flutter," Mathematical Problems in Engineering, vol. 2017, Article ID 6707092, 13 pages, 2017.

[18] F. Bakhtiari-Nejad and M. Nazari, "Nonlinear vibration analysis of isotropic cantilever plate with viscoelastic laminate," Nonlinear Dynamics, vol. 56, no. 4, pp. 325-356, 2009.

[19] K. M. Liew, K. C. Hung, and M. K. Lim, "Vibration of mindlin plates using boundary characteristic orthogonal polynomials," Journal of Sound and Vibration, vol. 182, no. 1, pp. 77-90, 1995.

[20] Z. X. Yang, Q. K. Han, Y. G. Chen et al., "Nonlinear harmonic response characteristics and experimental investigation of cantilever hard-coating plate," Nonlinear Dynamics, pp. 1-12, 2017.

[21] H.-H. Dai, J. K. Paik, and S. N. Atluri, “The global nonlinear galerkin method for the analysis of elastic large deflections of plates under combined loads: a scalar homotopy method for the direct solution of nonlinear algebraic equations," Computers, Materials and Continua, vol. 23, no. 1, pp. 69-99, 2011.

[22] H.-H. Dai, J. K. Paik, and S. N. Atluri, "The global nonlinear galerkin method for the solution of von karman nonlinear plate equations: an optimal and faster iterative method for the direct solution of nonlinear algebraic equations, using," Computers, Materials and Continua, vol. 23, no. 2, pp. 155-185, 2011.

[23] H. Dai, X. Yue, and S. Atluri, "Solutions of the von Kármán plate equations by a Galerkin method, without inverting the tangent stiffness matrix," Journal of Mechanics of Materials and Structures, vol. 9, no. 2, pp. 195-226, 2014.

[24] H. Dai, X. Yue, J. Yuan, and S. N. Atluri, "A time domain collocation method for studying the aeroelasticity of a two dimensional airfoil with a structural nonlinearity," Journal of Computational Physics, vol. 270, pp. 214-237, 2014.

[25] H. Dai, X. Wang, M. Schnoor, and S. N. Atluri, "Analysis of internal resonance in a two-degree-of-freedom nonlinear dynamical system," Communications in Nonlinear Science and Numerical Simulation, vol. 49, pp. 176-191, 2017.

[26] D. Younesian, H. Askari, Z. Saadatnia, and M. KalamiYazdi, "Frequency analysis of strongly nonlinear generalized Duffing oscillators using HE's frequency-amplitude formulation and HE's energy balance method," Computers \& Mathematics with Applications, vol. 59, no. 9, pp. 3222-3228, 2010.

[27] D. Younesian, H. Askari, Z. Saadatnia et al., "Free vibration analysis of strongly nonlinear generalized Duffing oscillators using He's variational approach and homotopy perturbation method," Nonlinear Science Letters A, vol. 2, no. 1, pp. 11-16, 2011.

[28] H. Askari, Z. Saadatnia, E. Esmailzadeh, and D. Younesian, "Multi-frequency excitation of stiffened triangular plates for 
large amplitude oscillations," Journal of Sound and Vibration, vol. 333, no. 22, pp. 5817-5835, 2014.

[29] M. Ye, Y. Sun, W. Zhang, X. Zhan, and Q. Ding, "Nonlinear oscillations and chaotic dynamics of an antisymmetric cross-ply laminated composite rectangular thin plate under parametric excitation," Journal of Sound and Vibration, vol. 287, no. 4-5, pp. 723-758, 2005.

[30] M. Sayed and A. Mousa, "Second-order approximation of angleply composite laminated thin plate under combined excitations," Communications in Nonlinear Science and Numerical Simulation, vol. 17, no. 12, pp. 5201-5216, 2012.

[31] W. Zhang, M. Zhao, and X. Guo, "Nonlinear responses of a symmetric cross-ply composite laminated cantilever rectangular plate under in-plane and moment excitations," Composite Structures, vol. 100, pp. 554-565, 2013.

[32] F. Alijani, F. Bakhtiari-Nejad, and M. Amabili, "Nonlinear vibrations of FGM rectangular plates in thermal environments," Nonlinear Dynamics, vol. 66, no. 3, pp. 251-270, 2011.

[33] F. Alijani and M. Amabili, "Non-linear dynamic instability of functionally graded plates in thermal environments," International Journal of Non-Linear Mechanics, vol. 50, pp. 109-126, 2013.

[34] C. Xue, E. Pan, Q. Han, S. Zhang, and H. Chu, "Non-linear principal resonance of an orthotropic and magnetoelastic rectangular plate," International Journal of Non-Linear Mechanics, vol. 46, no. 5, pp. 703-710, 2011.

[35] S. Shokrollahi and F. Bakhtiari-Nejad, "Limit cycle oscillations of swept-back trapezoidal wings at low subsonic flow," Journal of Aircraft, vol. 41, no. 4, pp. 948-953, 2004.

[36] W. Tian, Z. Yang, Y. Gu, and X. Wang, "Analysis of nonlinear aeroelastic characteristics of a trapezoidal wing in hypersonic flow," Nonlinear Dynamics, vol. 89, no. 2, pp. 1205-1232, 2017.

[37] W. Tian, Z. Yang, and Y. Gu, "Dynamic analysis of an aeroelastic airfoil with freeplay nonlinearity by precise integration method based on Padé approximation," Nonlinear Dynamics, vol. 89, no. 3, pp. 2173-2194, 2017. 


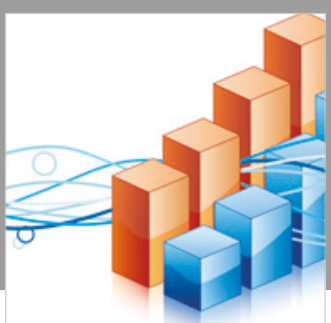

Advances in

Operations Research

\section{-n-m}
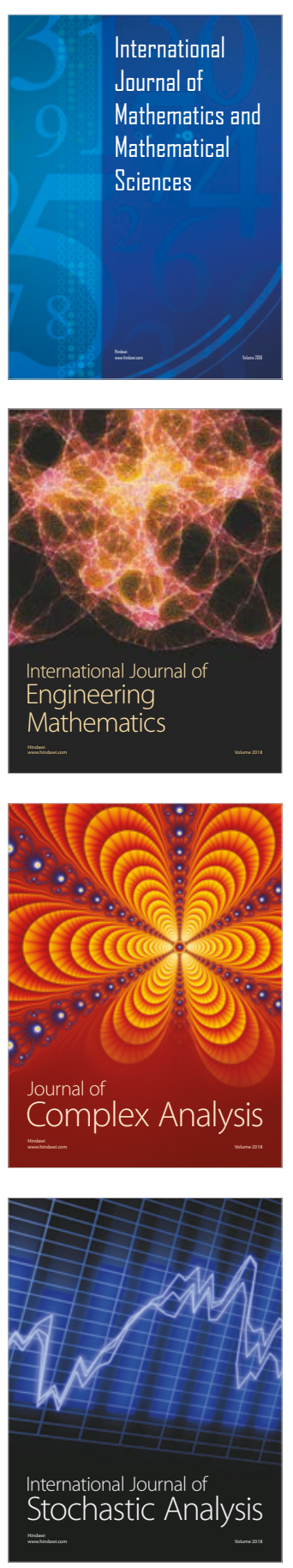
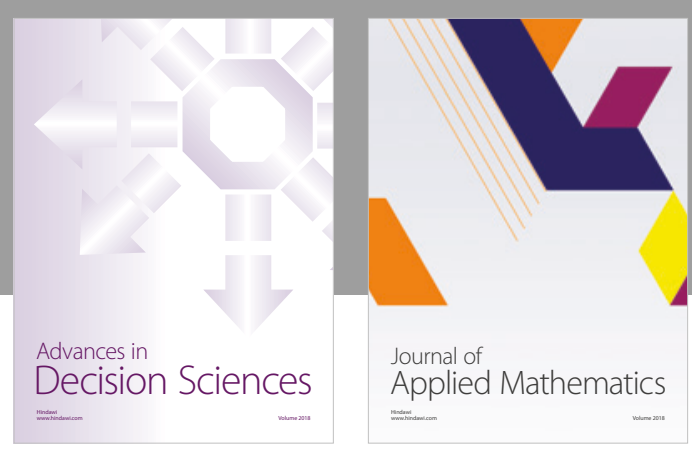

Journal of

Applied Mathematics
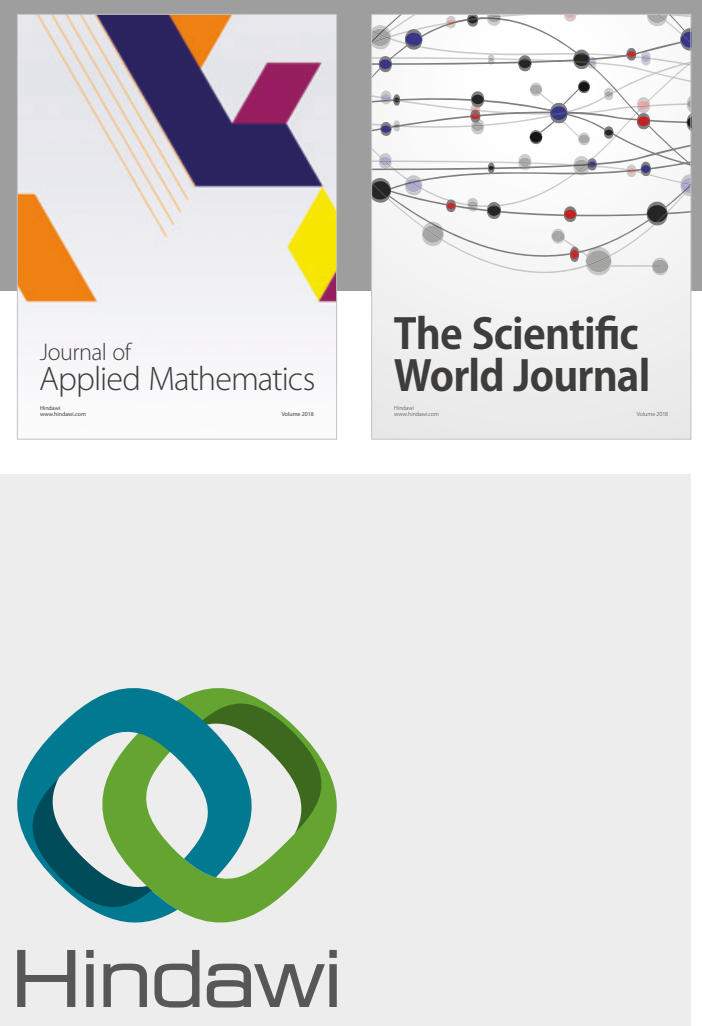

Submit your manuscripts at

www.hindawi.com

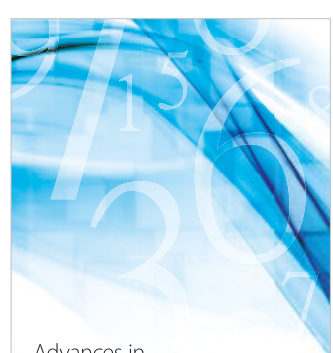

Advances in
Numerical Analysis
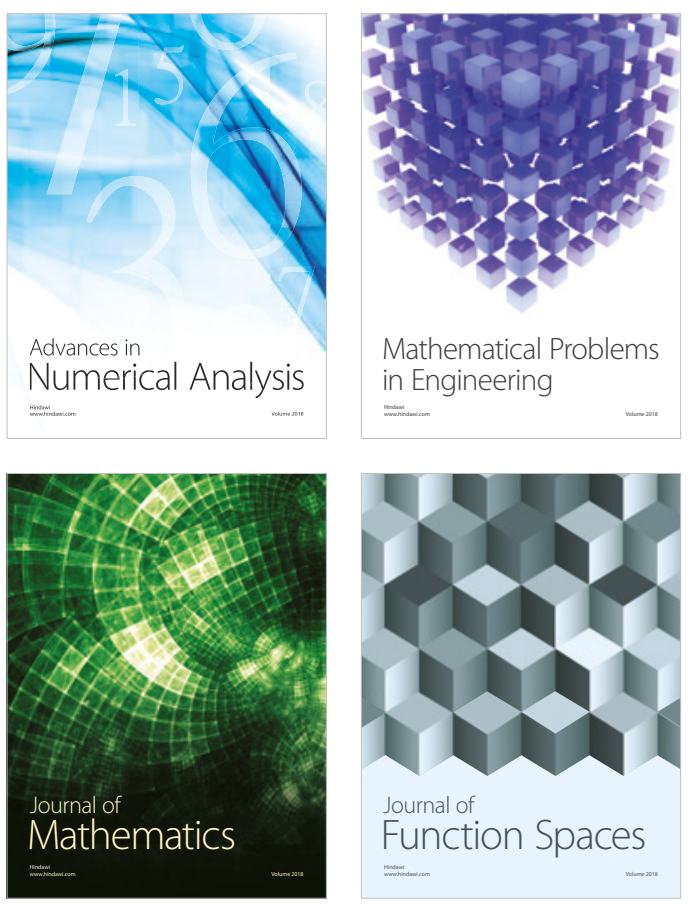

Mathematical Problems in Engineering

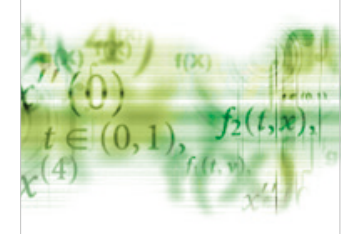

International Journal of

Differential Equations

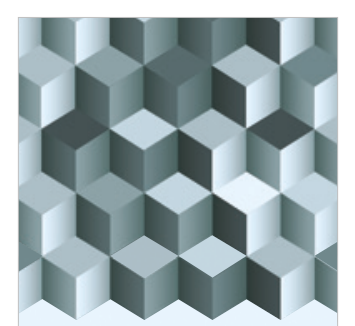

Journal of

Function Spaces

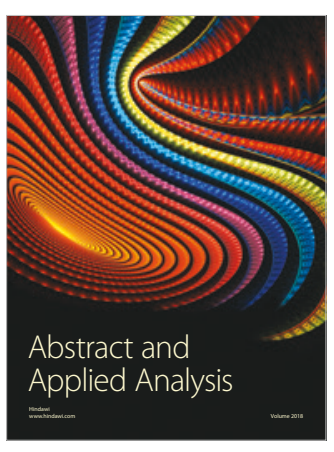

The Scientific

World Journal

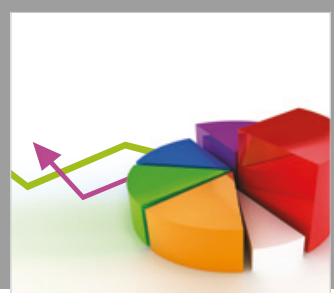

Journal of

Probability and Statistics
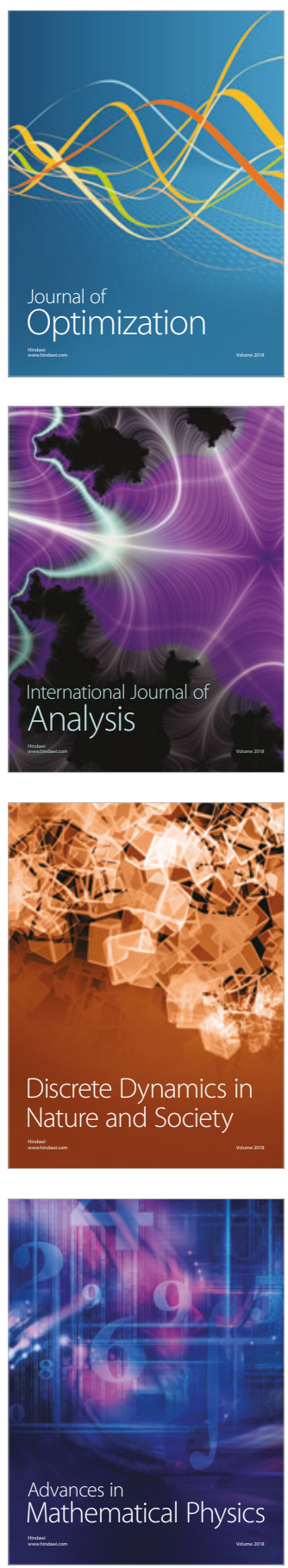